\title{
Maternal circulating miRNAs that predict infant FASD outcomes influence placental maturation
}

\author{
Alexander M Tseng ${ }^{1}$, Amanda H Mahnke ${ }^{1}$, Alan B Wells ${ }^{2,3}$, Nihal A Salem¹, Andrea M Allan ${ }^{4}$, Victoria HJ Roberts ${ }^{5}$, \\ Natali Newman ${ }^{6}$, Nicole AR Walter ${ }^{6}$, Christopher D Kroenke ${ }^{6}$, Kathleen A Grant ${ }^{6} \mathbb{0}$, Lisa K Akison ${ }^{7}$, Karen M Moritz \\ Christina D Chambers ${ }^{2,3}$, Rajesh C Miranda ${ }^{1} \mathbb{D}$, Collaborative Initiative on Fetal Alcohol Spectrum Disorders
}

\begin{abstract}
Prenatal alcohol exposure (PAE), like other pregnancy complications, can result in placental insufficiency and fetal growth restriction, although the linking causal mechanisms are unclear. We previously identified 11 gestationally elevated maternal circulating miRNAs ( following PAE. Here, we investigated whether these HEa miRNAs contribute to the pathology of PAE, by inhibiting trophoblast epithelial-mesenchymal transition (EMT), a pathway critical for placental development. We now report for the first time that PAE inhibits expression of placental pro-EMT pathway members in both rodents and primates, and that ${ }_{\text {HEamiRNAs collectively, but }}$ not individually, mediate placental EMT inhibition. HEamiRNAs collectively, but not individually, also inhibited cell proliferation and the EMT pathway in cultured trophoblasts, while inducing cell stress, and following trophoblast syncytialization, aberrant endocrine maturation. Moreover, a single intravascular administration of the pooled murine-expressed ${ }_{\mathrm{HEa}}$ miRNAs, to pregnant mice, decreased placental and fetal growth and inhibited the expression of pro-EMT transcripts in the placenta. Our data suggest that ${ }_{\text {HEa }}$ miRNAs collectively interfere with placental development, contributing to the pathology of PAE, and perhaps also, to other causes of fetal growth restriction.
\end{abstract}

DOI 10.26508/Isa.201800252 | Received 22 November 2018 | Revised 20 February 2019 | Accepted 21 February 2019 | Published online 4 March 2019

\section{Introduction}

Prenatal alcohol exposure (PAE) is common $(1,2,3)$. Between $1.1 \%$ and $5 \%$ of school children in the United States are conservatively estimated to have a fetal alcohol spectrum disorder (FASD) (4). Consequently, FASD, due to PAE, is the single largest cause of developmental disabilities in the United States and worldwide (5) and a comorbid factor in a number of other prevalent developmental neurobehavioral disabilities, including attention deficit/ hyperactivity and autism spectrum disorders (6).

PAE can result in decreased body weight, height, and/or head circumference in infants. Consequently, infant growth deficits are a cardinal diagnostic feature for fetal alcohol syndrome (7), which represents the severe end of the FASD continuum. However, although well recognized as a diagnostic feature, the mechanistic linkage between PAE and growth restriction remains unclear. In 2016, as part of our effort to identify maternal diagnostic biomarkers of the effect of PAE, we reported that elevated levels of 11 distinct miRNAs in maternal circulation during the second and third trimesters distinguished infants who were affected by in utero alcohol exposure (heavily exposed affected [HEa]) from those who were apparently unaffected at birth by PAE (heavily exposed unaffected [HEua]) or those who were unexposed (UE) (8). In that study, we predicted, based on bioinformatics analyses, that these ${ }_{\text {HEa miRNAs }}$ (MIMAT0004569 [hsa-miR-222-5p], MIMAT0004561 [hsa-miR-187-5p], MIMAT0000687 [hsa-miR-299-3p], MIMAT0004765 [hsa-miR-491-3p], MIMAT0004948 [hsa-miR-885-3p], MIMAT0002842 [hsa-miR-518f-3p], MIMAT0004957 [hsa-miR-760], MIMAT0003880 [hsa-miR-671-5p], MIMAT0001541 [hsa-miR-449a], MIMAT0000265 [hsa-miR-204-5p], and MIMAT0002869 [hsa-miR-519a-3p]) could influence signaling pathways crucial for early development, particularly the epithelialmesenchymal transition (EMT) pathway.

Placental development involves maturation of cytotrophoblasts at the tips of anchoring villi into invasive extravillous trophoblasts, as well as fusion of cytotrophoblasts into multinucleate, hormoneproducing syncytiotrophoblasts (9). Maturation into extravillous trophoblasts, which invade the maternal decidua and remodel the uterine spiral arteries into low-resistance high-flow vessels that enable optimal perfusion for nutrient and waste exchange, requires cytotrophoblasts to undergo EMT (10). Impaired placental EMT, as

\footnotetext{
${ }^{1}$ Department of Neuroscience and Experimental Therapeutics, Texas A\&M University Health Science Center, Bryan, TX, USA ${ }^{2}$ Clinical and Translational Research Institute, University of California San Diego, San Diego, CA, USA ${ }^{3}$ Department of Pediatrics, University of California San Diego, San Diego, CA, USA ${ }^{4}$ Department of Neurosciences, University of New Mexico, Albuquerque, NM, USA ${ }^{5}$ Division of Reproductive and Developmental Sciences, Oregon National Primate Research Center, Oregon Health \& Science University, Portland, OR, USA ${ }^{6}$ Division of Neuroscience, Oregon National Primate Research Center, Oregon Health \& Science University, Portland, OR, USA ${ }^{7}$ Child Health Research Centre and School of Biomedical Sciences, The University of Queensland, Brisbane, Australia
} 
well as orchestration of the opposing mesenchymal-epithelial transition pathway, has been found in conditions resulting from placental malfunction, primarily preeclampsia $(11,12,13,14,15,16)$. Although there have been no previous studies directly investigating the effects of PAE on placental EMT, a rodent study demonstrated that PAE, during a broad developmental window, reduced the number of invasive trophoblasts within the mesometrial triangle, a region of the uterine horn directly underlying the decidua (17). Furthermore, both human and rodent studies have found PAE disrupts placental morphology and interferes with cytotrophoblast maturation, as with preeclampsia $(18,19,20,21)$. Disrupted trophoblast maturation, seen in these conditions, is associated with aberrant expression of placental hormones, primarily human chorionic gonadotropin (hCG) $(22,23,24,25)$.

Our study is the first to report that PAE interferes with expression of core placental EMT pathway members. Using rodent and primate models of gestation, as well as complementary miRNA overexpression and knockdown studies in vitro, we also provide evidence

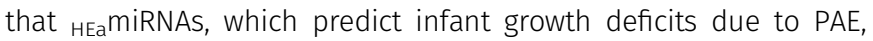
collectively but not individually, mediate PAE's effects on placental EMT through their effects on cytotrophoblast maturation and cellular stress. In a mouse model of pregnancy, a single combined exposure

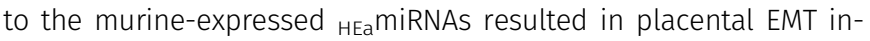
hibition and diminished placental and fetal growth. Collectively, these data suggest that elevated HEamiRNAs may represent an emergent maternal stress response that triggers fetal growth restriction, although subgroups of ${ }_{\mathrm{HEa}}$ miRNAs may compete to protect against the loss of EMT. Moreover, most members of the group of
HEa miRNAs have also been implicated in other placental insufficiency and growth restriction syndromes, giving rise to the possibility that growth restriction syndromes may share common etiological mediators.

\section{Results}

HEamiRNAs are implicated in placental-associated pathologies

Given our prediction that ${ }_{\mathrm{HEa}}$ miRNAs interfere with signaling pathways governing fetal and placental development (8), we conducted a literature review of reports on $\mathrm{HEa}_{\mathrm{m}}$ miRNA levels in gestational pathologies caused by poor placentation (26, 27, 28). Surprisingly, placental and plasma levels of 8 of $11_{\mathrm{HEa}}$ miRNAs were significantly dysregulated in one or more of these gestational pathologies with expression of the majority of these eight miRNAs altered in both fetal growth restriction and preeclampsia (Fig 1A) $(29,30,31,32,33,34,35$, $36,37,38,39,40,41,42,43,44,45,46,47,48,49)$, both of which are characterized by poor placental invasion $(50,51,52,53,54,55,56)$.

\section{HEamiRNAs explain variance in infant growth outcomes due to PAE}

Given the association of individual HEamiRNAs with gestational pathologies, we sought to determine if circulating HEamiRNA levels could explain the variance in sex and gestational age-adjusted neonatal height, weight, and head circumference in our Ukrainian birth cohort, which are growth measures sensitive to in utero

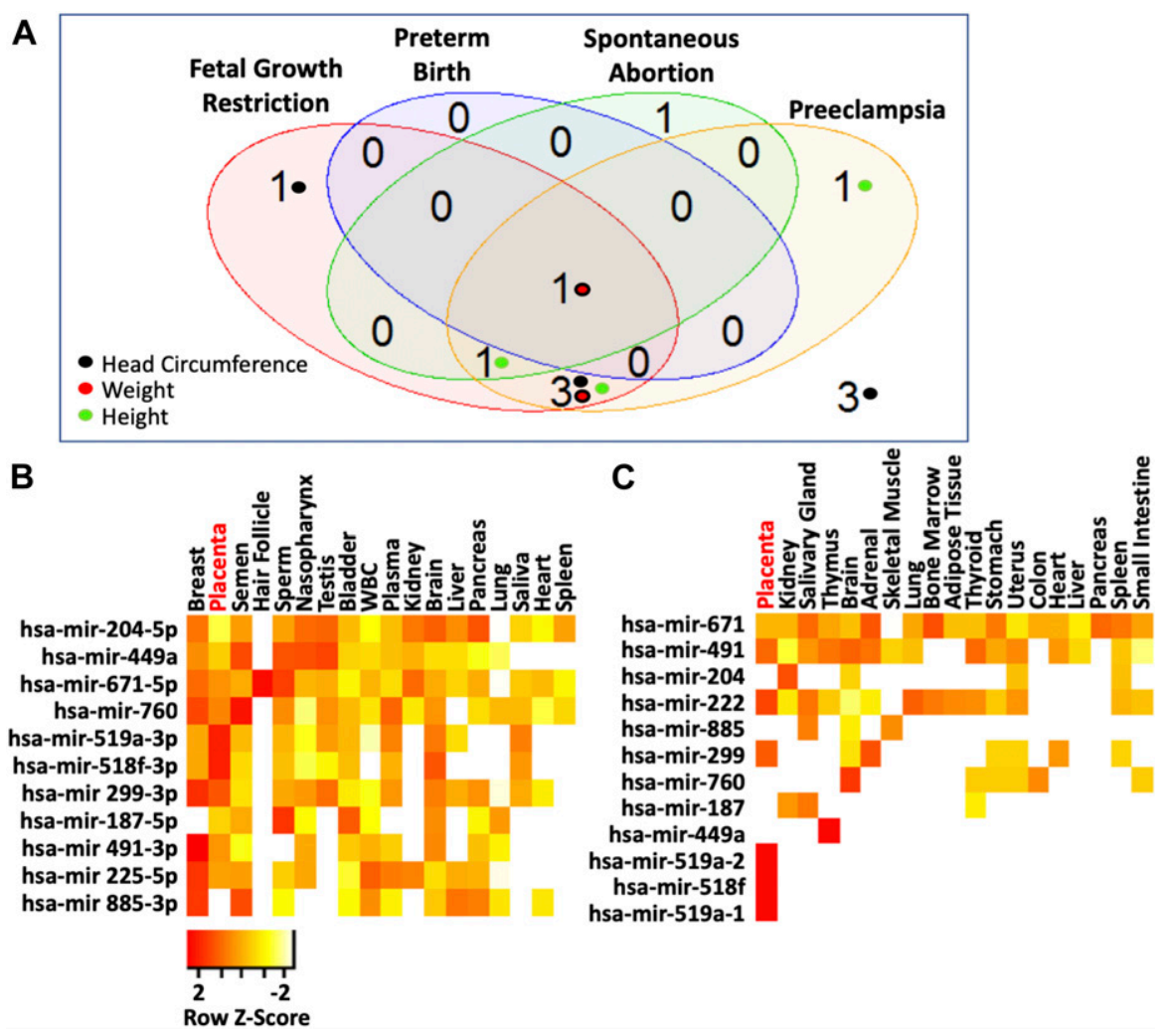

Figure 1. HEamiRNAs are placentally enriched and associated with gestational pathologies.

(A) Venn diagram on number of ${ }_{\mathrm{HEa}}$ miRNAs reported to be associated with different gestational pathologies. Inset colored circles represent the corresponding sex and gestational age-adjusted growth parameters these miRNAs were correlated with. Of the 22 studies queried, $11(50 \%)$ used unbiased screenings for miRNA expression. (B, C) Heat map of mature ${ }_{\mathrm{HEa}}$ miRNA expression (B) and pri- ${ }_{\text {HEa }}$ miRNA expression (C) across different tissues resulting from secondary analysis of publicly available RNA-sequencing data. Legend depicts row-centered Z-score. 
Table 1. HEamiRNAs are significantly correlated with independent measures of infant size.

\begin{tabular}{|c|c|c|c|c|c|c|c|c|c|c|c|}
\hline \multirow{2}{*}{ MIMAT no. } & \multirow{2}{*}{ miRNA } & \multirow{2}{*}{ Trimester } & \multicolumn{3}{|l|}{ Weight } & \multicolumn{3}{|l|}{ Height } & \multicolumn{3}{|c|}{ Head circumference } \\
\hline & & & Sig. & $\mathrm{R}^{2}$ & $\rho$ & Sig. & $\mathrm{R}^{2}$ & $\rho$ & Sig. & $\mathrm{R}^{2}$ & $\rho$ \\
\hline MIMAT0004569 & hsa-miR-222-5p & 2 & 0.821 & 1.224 & -0.051 & 0.066 & 9.572 & -0.179 & 0.8 & 1.732 & -0.104 \\
\hline MIMAT0004561 & hsa-miR-187-5p & 2 & 0.462 & 6.347 & 0.068 & 0.17 & 12.607 & -0.074 & 0.134 & 10.903 & 0.103 \\
\hline MIMAT0000687 & hsa-miR-299-3p & 2 & 0.552 & 1.113 & 0.029 & 0.069 & 9.299 & -0.203 & $0.036^{a}$ & 8.65 & 0.1 \\
\hline MIMAT0004765 & hsa-miR-491-3p & 2 & 0.172 & 3.61 & 0.112 & 0.849 & 2.033 & -0.055 & $0.024^{a}$ & 12.529 & 0.156 \\
\hline МIMAT0004948 & hsa-miR-885-3p & 2 & 0.142 & 4.227 & -0.174 & $0.044^{a}$ & 7.667 & -0.231 & 0.59 & 1.36 & -0.115 \\
\hline MIMAT0002842 & hsa-miR-518f-3p & 2 & 0.246 & 2.517 & 0.134 & 0.918 & 2.134 & -0.118 & $0.007^{b}$ & 14.561 & 0.219 \\
\hline MIMAT0004957 & hsa-miR-760 & 2 & 0.059 & 6.314 & 0.195 & 0.22 & 4.096 & 0.079 & 0.055 & 10.158 & 0.195 \\
\hline MIMAT0003880 & hsa-miR-671-5p & 2 & 0.123 & 7.24 & 0.11 & 0.578 & 5.264 & -0.031 & 0.073 & 10.794 & 0.107 \\
\hline MIMAT0001541 & hsa-miR-449a & 2 & 0.101 & 11.584 & 0.104 & 0.718 & 5.851 & -0.072 & 0.173 & 10.036 & 0.068 \\
\hline MIMAT0000265 & hsa-miR-204-5p & 2 & $0.026^{a}$ & 12.377 & 0.184 & 0.272 & 4.973 & 0 & 0.131 & 7.095 & 0.108 \\
\hline MIMAT0002869 & hsa-miR-519a-3p & 2 & $0.034^{a}$ & 7.975 & 0.153 & 0.403 & 6.83 & -0.012 & 0.093 & 8.181 & 0.096 \\
\hline МІМАТ0004569 & hsa-miR-222-5p & 3 & 0.875 & 0.993 & -0.046 & $0.018^{\mathrm{a}}$ & 10.709 & -0.196 & 0.577 & 4.696 & -0.01 \\
\hline MIMAT0004561 & hsa-miR-187-5p & 3 & 0.538 & 2.055 & 0.049 & 0.37 & 2.029 & -0.109 & 0.784 & 3.697 & 0.002 \\
\hline MIMAT0000687 & hsa-miR-299-3p & 3 & 0.511 & 0.762 & 0.005 & 0.514 & 1.769 & -0.072 & 0.87 & 3.786 & -0.077 \\
\hline MIMAT0004765 & hsa-miR-491-3p & 3 & 0.824 & 3.165 & -0.028 & 0.2 & 12.122 & -0.121 & 0.747 & 4.188 & -0.081 \\
\hline MIMAT0004948 & hsa-miR-885-3p & 3 & 0.807 & 0.148 & 0.029 & 0.102 & 4.686 & -0.156 & 0.376 & 5.009 & 0.032 \\
\hline MIMAT0002842 & hsa-miR-518f-3p & 3 & 0.515 & 2.099 & 0.109 & 0.421 & 1.715 & 0.016 & 0.245 & 7.917 & 0.152 \\
\hline MIMAT0004957 & hsa-miR-760 & 3 & 0.368 & 1.396 & 0.141 & 0.761 & 0.716 & -0.022 & 0.207 & 6.052 & 0.172 \\
\hline MIMAT0003880 & hsa-miR-671-5p & 3 & 0.055 & 8.715 & 0.155 & 0.367 & 3.521 & -0.133 & 0.076 & 8.196 & 0.15 \\
\hline MIMAT0001541 & hsa-miR-449a & 3 & 0.995 & 0.085 & -0.06 & 0.982 & 0.678 & -0.151 & $0.026^{a}$ & 12.022 & 0.135 \\
\hline MIMAT0000265 & hsa-miR-204-5p & 3 & $0.019^{a}$ & 11.872 & 0.23 & 0.206 & 5.589 & 0.022 & $0.002^{\mathrm{b}}$ & 18.683 & 0.319 \\
\hline MIMAT0002869 & hsa-miR-519a-3p & 3 & 0.391 & 2.82 & 0.043 & 0.302 & 5.917 & -0.151 & 0.106 & 9.286 & 0.118 \\
\hline
\end{tabular}

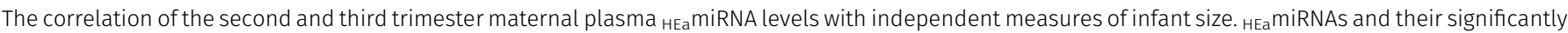
correlated sex and gestational age-adjusted growth parameters appear in bold. $\mathrm{R}^{2}$ is expressed as the percentage $(\times 100)$ of variance explained.

${ }^{\mathrm{a}} P<0.05$.

${ }^{\mathrm{b}} \mathrm{P}<0.01$.

environment (57). We found that eight of the HEamiRNAs each significantly explained between $7 \%$ and $19 \%$ of infant variation in these growth measures (Table 1). Furthermore, seven of these miRNAs were also associated with fetal growth restriction and preeclampsia as identified by our literature review (Fig 1A). Interestingly, a multivariate statistical regression model that accounted for levels of all 11 HEamiRNAs together, explained a far greater proportion of infant variance, between $24 \%$ and $31 \%$, in all three growth measures than accounting for them individually (Table S1),

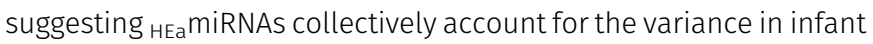
growth outcomes.

HEa miRNAs are transcribed preferentially in the placenta

Data extracted from publicly available gene expression profiling datasets (58) show that HEamiRNAs and their unprocessed precursor transcripts, HEapri-miRNAs, are enriched in placenta compared with other tissues, suggesting that the placenta itself transcribes these miRNAs and may be a significant contributory tissue to maternal circulating $\mathrm{HEa}_{\mathrm{a}}$ miRNAs (Fig 1B and C). Moreover, because HEamiRNAs are also associated with gestational patholo-

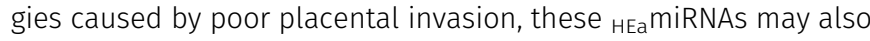
contribute to the placental response to PAE. We, therefore, assessed in rodent and primate models, whether PAE could result

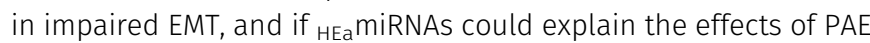
on placental EMT-associated gene expression.

\section{HEa miRNAs moderate placental EMT impairment in PAE models}

EMT, in trophoblasts, is characterized by the disappearance of epithelial markers such as E-Cadherin and the appearance of mesenchymal markers such as the intermediate filament, vimentin, a process that is controlled by the expression of key mesenchymal determination transcription factors, Snail 1 and 2 and TWIST, as extensively described $(10,14,15,59,60,61,62)$. These five markers have been used to assess EMT in a variety of model systems, so our studies used these markers to assess the effects of alcohol and HEamiRNAs on trophoblast EMT.

In the first analysis, using a murine model of PAE that mimicked moderate to binge-type alcohol consumption throughout early and 
mid-pregnancy, we fractionated GD14 placenta into three zones: the cytotrophoblast- and syncytiotrophoblast-rich labyrinth zone, the glycogen- and spongiotrophoblast-rich junctional zone, and the decidual zone comprising the endometrial contribution to the placenta (Fig 2A). Multivariate analysis of variance (MANOVA) for expression of these five core genes in the EMT pathway within
A

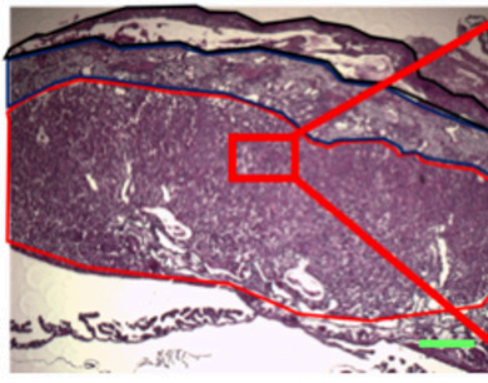

B

Mouse

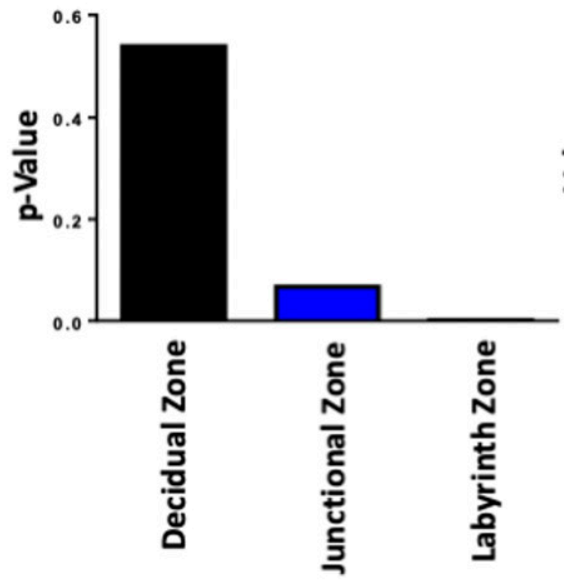

C
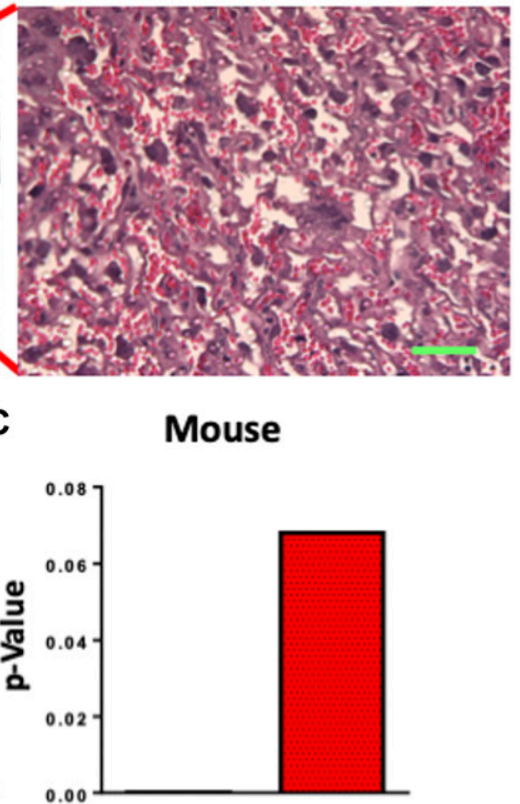

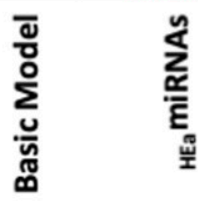

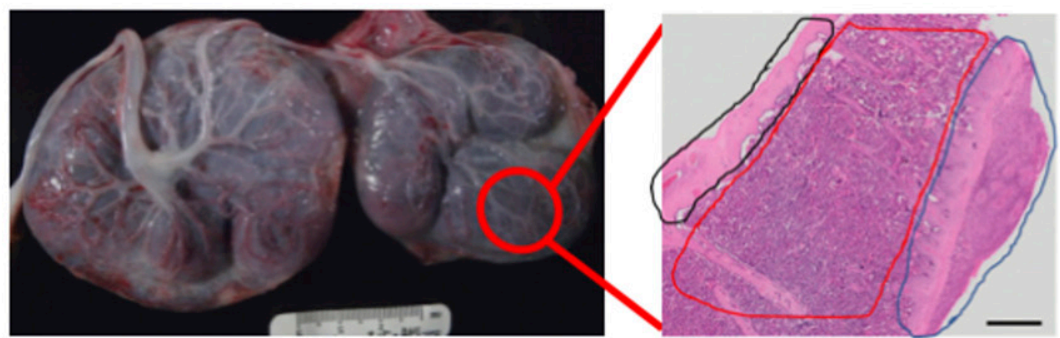

$\mathbf{E}$

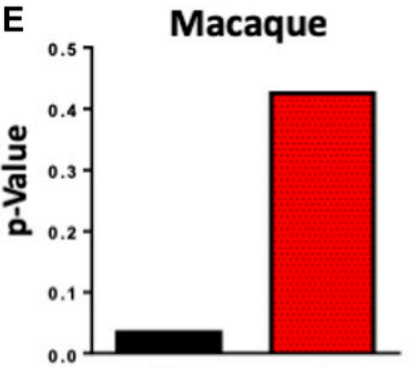

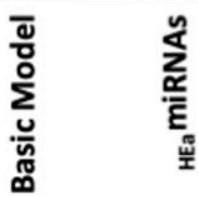

$\mathbf{F}$

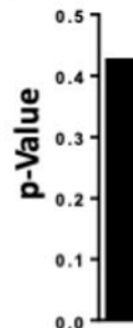

Macaque

Figure 2. miRNAs mediate the effect of PAE on EMT pathway members in mouse and macaque placentas. (A) Histological image of GD14 mouse placenta. Outlined in red is the labyrinth zone, blue is the junctional zone, and black is the decidual zone with the scale bar (green) demarcating $200 \mu \mathrm{m}$. Inset is a high-magnification image of the labyrinth zone with the scale bar (green) demarcating $50 \mu \mathrm{m}$. (B) MANOVA of gene expression of core EMT pathway members in different regions of the mouse placenta in control and PAE mice $(n=29$ samples). (c) MANCOVA of gene expression of core EMT pathway members in the mouse placental labyrinth zone before (Basic Model) and after accounting for the

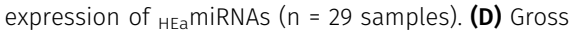
anatomy photograph of the primary (left) and secondary (right) lobes of a GD135 macaque placenta. Outlined in red is an individual cotyledon from the secondary lobe. Inset is a full thickness hematoxylin and eosin-stained histological section of a representative cotyledon with the fetal membranes outlined in black, villous tissue outlined in red. and maternal decidua in blue. Ruler is $3 \mathrm{~cm}$ and scale bar (black) is $2 \mathrm{~mm}$. (E) MANCOVA of gene expression of core EMT pathway members in placental cotyledons of PAE and control macaques, accounting for the expression of HEamiRNAs collectively ( $n=23$ samples). ( $F$ ) MANCOVA of gene expression of core EMT pathway members in macaque placentas after accounting for expression of HEamiRNAs individually ( $n=23$ samples). 
placental trophoblasts revealed a significant effect of ethanol exposure on EMT pathway member expression selectively within the labyrinth zone (Pillai's trace statistic, $F_{(5,21)}=6.85, P<0.001$, Fig 2B) but not within the junctional or decidual zones. Post hoc univariate ANOVA indicated ethanol exposure specifically elevated $\mathrm{CDH} 1$ $\left(F_{(1,25)}=7.452, P=0.011\right)$, which encodes epithelial E-Cadherin, whereas expression of the pro-mesenchymal transcription factor SNAI1, which encodes Snail1, was significantly reduced $\left(F_{(1,25)}=21.022, P=\right.$ 0.0001). We also observed a significant interaction between fetal sex and PAE on expression of SNAI2, which encodes Snail2 $\left(F_{(1,25)}=2.18\right.$, $P=0.047)$ and a trend towards decreased expression of the terminal mesenchymal marker VIM (vimentin, $F_{(1,25)}=2.749, P=0.11$ ), whereas there was no effect on TWIST expression (Fig 3A-E). Consistent with our gene expression data, E-Cadherin protein levels were significantly elevated in the labyrinth zone of PAE placenta $\left(F_{(1,24)}=31.63\right.$, $P=0.0005$ ), whereas not in the junctional or decidual zones (Figs $3 F$ and S1A and B). However, when we controlled for expression of the eight mouse homologs of HEamiRNAs as a covariate, using multivariate analysis of covariance (MANCOVA), ethanol's effect on EMT became marginally nonsignificant (Pillai's trace, $F_{(5,21)}=2.713, P=$ 0.068) (Fig 2C), suggesting that these miRNAs partially mediate effects of PAE on EMT pathway members in mice. Interestingly, PAE
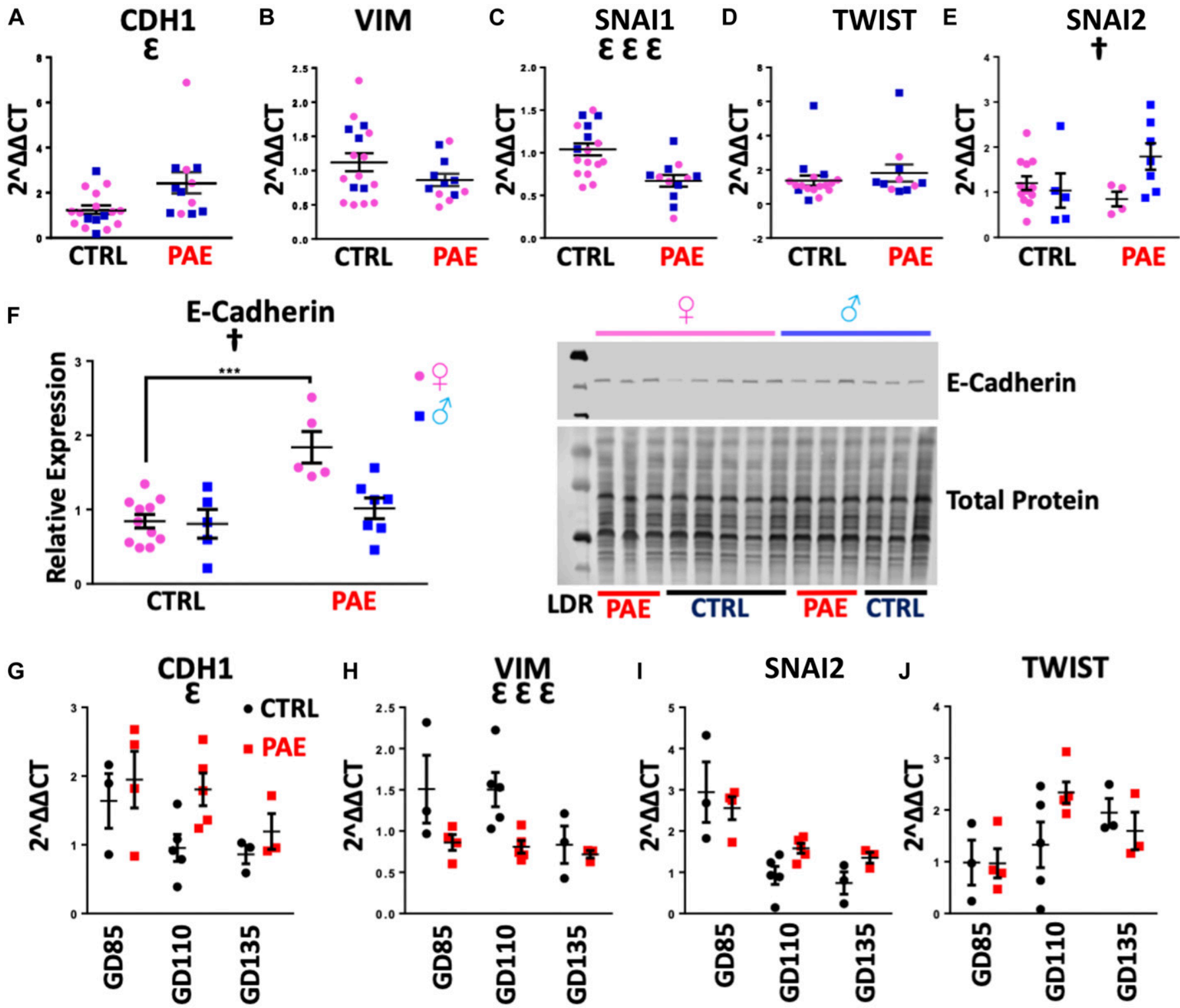

TWIST

Figure 3. PAE interferes with the EMT pathway in mouse and macaque placentas.

(A-E) Expression of CDH1 (A), VIM (B), SNAI1 (C), TWIST (D), and SNAI2 (E) in the placental labyrinth zone of PAE and control mice ( $\mathrm{n}=5-12$ samples per group). (F) Densitometric quantification of E-Cadherin expression in the labyrinth zone of PAE and control mice as well as representative blot of E-Cadherin expression and total protein expression (right, $n=5-12$ samples per group). (G-J) Expression of CDH1 (G), VIM (H), SNAI2 (I), and TWIST (J) transcripts in PAE and control macaque placental cotyledons ( $n=3-5$ samples per group). Results are expressed as the mean \pm SEM, LDR $=$ molecular weight ladder; ANOVA: significant main effect of PAE $\left({ }^{\varepsilon} P<0.05\right.$, ${ }^{\varepsilon \varepsilon \varepsilon_{P}}<$ 0.001), significant interaction effect (sex by PAE, $\left[{ }^{\dagger} P<0.05\right]$ ). For post hoc analysis, ${ }^{\star \star \star} P<0.001$ by Tukey's HSD. 
limited to the periconceptional period in rats also influenced expression of EMT core transcripts (Figs S2B and S3A-E).

To determine if PAE's effects on EMT pathway members in placenta are broadly conserved throughout mammalian evolution, we adopted a nonhuman primate (macaque) model of moderate to binge-type alcohol consumption. Placental tissues were isolated from GD85, GD110, and GD135 placenta (Fig 2D), which spans the human equivalent of the mid-second to mid-third trimester (Fig S2C). There was a significant effect of ethanol exposure on expression of core EMT mRNA transcripts by MANOVA (Pillai's trace statistic, $F_{(4,9)}=4.229 P=0.045$, Fig 3B). Consistent with our findings in mouse, post hoc univariate ANOVA indicated that in primate placenta, ethanol exposure significantly increased $C D H 1$ expression $\left(F_{(1,12)}=4.866, P=0.048\right)$, whereas VIM expression was significantly reduced $\left(F_{(1,12)}=12.782, P=0.0004\right)$, suggesting that, as in the mouse, PAE also impairs EMT in the primate placenta. Interestingly, there was no effect on SNAI2 or TWIST expression (Fig 3G-J). As in mice, accounting for expression of HEamiRNAs together as a covariate abolished the significant effect of PAE on EMT, although to a greater degree than mice (Pillai's trace, $F_{(1,1)}=1.605, P=0.425$, Fig $2 E$ ). Interestingly, accounting for expression of individual $\mathrm{HEa}_{\mathrm{miRNAs}}$ did not explain the effects of PAE on placental EMT, suggesting that HEamiRNAs act in concert to mediate the effect of PAE on EMT in the primate placenta (Fig 2F).

Collectively, our data suggest PAE-induced impairment of EMT in the trophoblastic compartment of placentae is conserved between rodents and nonhuman primates and that HEamiRNAs, particularly in primates, may moderate the effect of PAE on placental EMT. Consequently, subsequent studies focused on the collective role of $\mathrm{HEa}_{\mathrm{miRNAs}}$, either on basal or on alcohol-influenced placental trophoblast growth, invasion, and the maturation of physiological function.

\section{HEamiRNAs impair EMT in a model of human cytotrophoblasts}

To investigate whether $\mathrm{HEa}_{\mathrm{m}}$ miRNAs collectively interfere with the EMT pathway, as suggested by our in vivo data, we examined the effects of transfecting $\mathrm{HEa}_{\mathrm{m}}$ miRNA mimics and antagomirs into BeWO cytotrophoblasts (Fig 4A). We initially overexpressed each of the 11 HEa miRNAs individually, to determine whether any of them could influence the EMT pathway. We did not observe any significant effects (Fig S4), consistent with our findings in the primate PAE model that individual miRNAs did not explain the effects of ethanol on EMT. In contrast, transfection of pooled ${ }_{\mathrm{HEa}}$ miRNAs into cytotrophoblasts significantly increased $\mathrm{CDH} 1$ expression $\left(\mathrm{F}_{(1,36)}=30.08\right.$, $P<0.0001)$. Interestingly, expression of the pro-mesenchymal transcription factors TWIST and SNAI1 were also significantly reduced, but only in the context of concomitant $320 \mathrm{mg} / \mathrm{dl}$ ethanol treatment, pointing to an interaction effect between ${ }_{\mathrm{HEa}}$ miRNAs and ethanol $\left(F_{(1,36)}=5.650\right.$ and 5.146, respectively, $P=0.023$ and $P=0.029$, Fig 4B-E). Consistent with our qPCR data, transfection of $\mathrm{HEa}_{\mathrm{miRNAS}}$ also significantly increased $\mathrm{E}$-Cadherin protein expression $\left(\mathrm{F}_{(1,20)}=\right.$ 33.86, $P<0.0001$, Fig 4F). We were unable to detect SNAI2 transcript expression or vimentin protein expression in these cells, consistent with previous reports (63).

We next sought to determine if more restricted subsets of

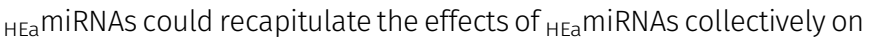
EMT. Thus, we overexpressed hsa-miR-222-5p and hsa-miR-519a-3p, which are implicated in preeclampsia and fetal growth restriction, as well as hsa-miR-885-3p, hsa-miR-518f-3p, and hsa-miR-204-5p, which are implicated in preeclampsia, fetal growth restriction, and spontaneous abortion or preterm labor (Fig S5A). In contrast to the collective action for all $\mathrm{HEa}_{\mathrm{m}}$ miRNAs, exposure to each of these pools resulted in significant decreases in $C D H 1$ expression $\left(F_{(2,12)}=20.12\right.$, $P=0.0001)$. The pool including hsa-miR-885-3p, hsa-miR-518f-3p, and hsa-miR-204-5p also significantly increased Snai1 SNA/1 $\left(F_{(2,12)}=\right.$ 4.604, $P=0.0328$; Dunnett's post hoc $P=0.0497$, Fig S5B-E). These data suggest that ${ }_{H E a}$ miRNAs include subgroups of miRNAs that have the potential to partly mitigate the effects of elevating the entire pool. However, the potential protective effects of these subgroups are masked by the collective function of the entire group of HEa miRNAS.

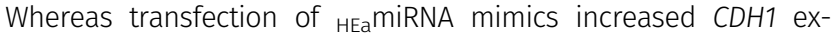
pression, transfection of pooled antagomirs to $\mathrm{HEa}$ miRNAs significantly reduced $\mathrm{CDH} 1$ expression, only in the context of $320 \mathrm{mg} / \mathrm{dl}$ ethanol co-

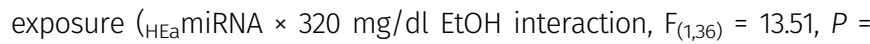
0.0008; post hoc Tukey's honest significance difference (HSD), $P=0.005$, Fig 4G). However, expression of TWIST was also decreased with ethanol co-exposure, and there was no significant difference in E-Cadherin protein expression relative to the control (Fig $4 \mathrm{H}-\mathrm{K}$ ). Thus, our data suggest that increasing HEamiRNA levels impairs EMT pathway members in cytotrophoblasts, whereas inhibiting their action has a more restricted effect on EMT pathway members.

\section{HEamiRNAs impair EMT in a model of human extravillous trophoblasts}

We next investigated the effect of ${ }_{\text {HEa }}$ miRNAs on EMT in HTR-8/ SVneo extravillous trophoblast-type cells (Fig 5A). Transfecting pooled ${ }_{\mathrm{HEa}}$ miRNA mimics into extravillous trophoblasts significantly decreased VIM expression $\left(F_{(1,36)}=28.43, P<0.0001\right)$. Expression of pro-mesenchymal transcription factor SNAI2 was also reduced $\left(F_{(1,36)}=64.88, P<0.0001\right)$. As with cytotrophoblasts, expression of SNAI1 and TWIST were reduced only with $320 \mathrm{mg} / \mathrm{dl}$ ethanol coexposure ( ${ }_{\text {HEa }}$ miRNA $\times 320 \mathrm{mg} / \mathrm{dl}$ EtOH interaction, $\mathrm{F}_{(1,36)}=4.21$ and $5.18, P=0.048$ and 0.029 , respectively; post hoc Tukey's HSD, $P=0.027$ and $P<0.0001$, respectively, Fig 5B-E). Consistent with our qPCR data, vimentin protein expression was also significantly reduced $\left(F_{(1,20)}=9.535, P=0.006\right.$, Fig 5F). Interestingly, there was also a main effect of alcohol exposure on decreasing vimentin protein expression $\left(F_{(1,20)}=7.303, P=0.014\right)$. We were unable to detect expression of $\mathrm{CDH} 1$ transcript, or its $\mathrm{E}$-Cadherin protein product, in extravillous trophoblasts, consistent with previous reports (63).

In contrast to $\mathrm{HEa}_{\mathrm{m}}$ miRNA mimics, transfecting pooled antagomirs significantly increased VIM expression $\left(F_{(1,35)}=42.56, P<0.0001\right)$. Likewise, antagomir transfection increased expression of SNAI2 in the context of $320 \mathrm{mg} / \mathrm{dl}$ ethanol co-exposure and SNAl1 under basal conditions $\left(_{\text {HEa }}\right.$ miRNA $\times 320 \mathrm{mg} / \mathrm{dl}$ Etoh interaction, $\mathrm{F}_{(1,35)}=$ 10.31 and 4.86, $P=0.01$ and $P=0.034$, respectively; post hoc Tukey's HSD, $P<0.0001$, Fig 5G-J). Despite our qPCR data, we did not observe significant differences in vimentin protein expression between treatment groups (Fig 5K). Collectively, our data indicate that in-

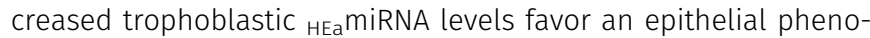
type, whereas inhibiting their action promotes a mesenchymal phenotype. 


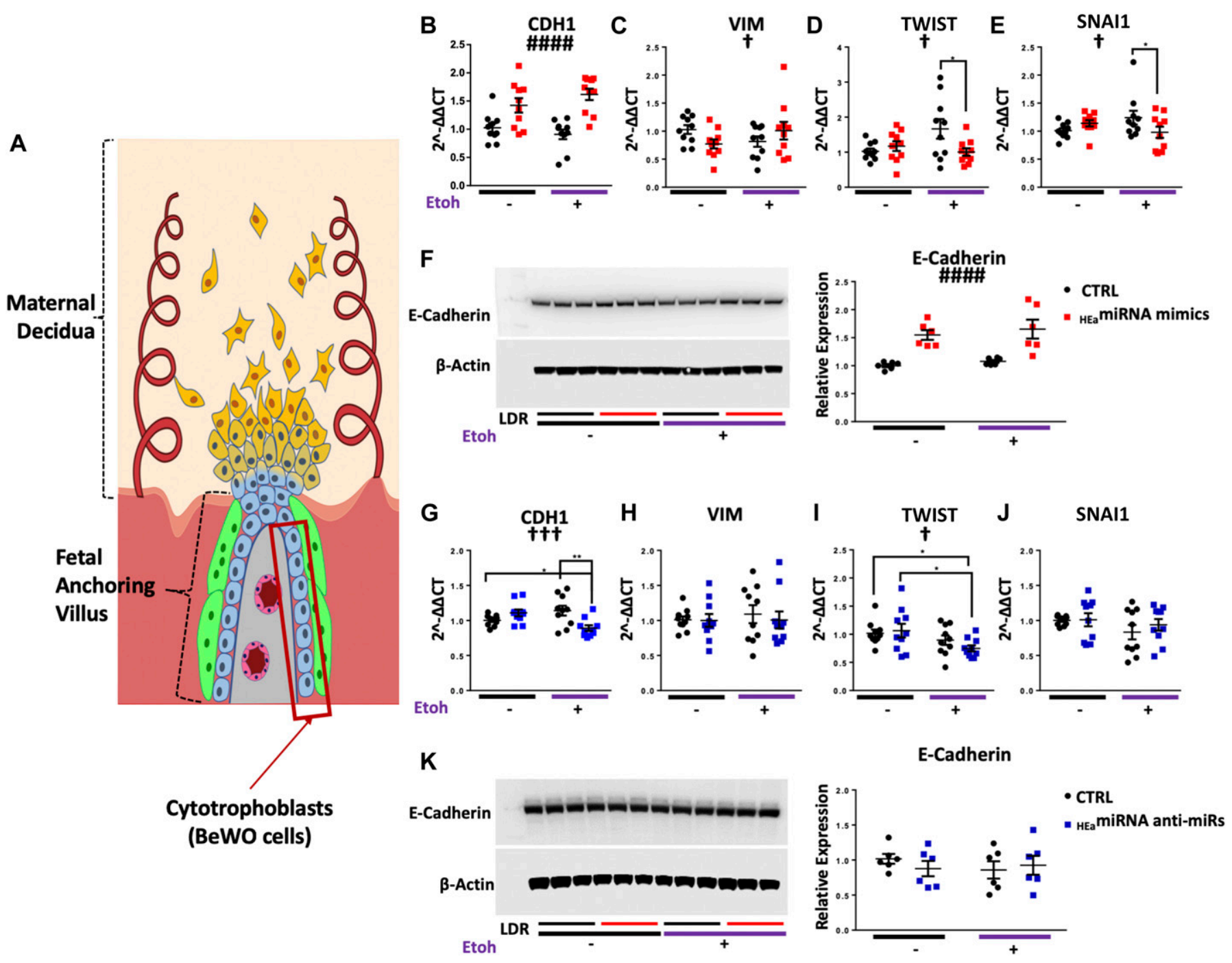

Figure 4. HEamiRNAs interfere with the EMT pathway in BeWO cytotrophoblasts.

(A) Diagram of a placental anchoring villous and maternal decidua with the boxed area denoting cytotrophoblasts. (B-F) Expression of CDH1 (B), VIM (C), TWIST (D), and SNAI7 (E) transcripts and densitometric quantification of E-Cadherin protein levels (F) in BeWO cytotrophoblasts following HEamiRNA or control miRNA overexpression with or without concomitant $320 \mathrm{mg} / \mathrm{dl}$ ethanol exposure. (G-K) Expression of CDH1 (G), VIM (H), TWIST (I), and SNAl1 transcripts (J) and densitometric quantification of E-Cadherin protein levels $(\mathrm{K})$ in BeWO cytotrophoblasts following HEamiRNAs or control hairpin inhibitor transfection with or without concomitant $320 \mathrm{mg} / \mathrm{dl}$ ethanol exposure. Results are expressed as the mean \pm SEM, LDR = molecular weight ladder, $n=10$ samples per group; ANOVA: significant main effect of $\mathrm{HEa}_{\mathrm{miRNA}}$ transfection ( ${ }^{\# \# \#+1} P<0.0001$ ), significant interaction effect (

\section{Antagomirs prevent ${ }_{\text {HEa }}$ miRNAs' inhibition of EMT}

We next investigated if pretreating cytrophoblasts with pooled HEamiRNA antagomirs could prevent inhibition of the EMT pathway caused by transfecting HEamiRNA mimics. Pretreatment of cyto-

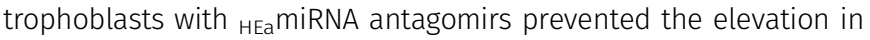
CDH1 caused by transfection with ${ }_{\mathrm{HEa}}$ miRNA mimics (post hoc Tukey's HSD, $n=10$ samples per group, $P=0.004$ ). Likewise, pre-transfection with ${ }_{\mathrm{HEa}}$ miRNA antagomirs also prevented $\mathrm{HEa}_{\mathrm{m}}$ miRNA mimic-induced reduction of SNAl1 and VIM expression (post hoc Tukey's HSD, $n=10$ samples per group, $P=0.007$ and $P<0.0001$, respectively) (Fig 6A-D).

As with cytotrophoblasts, pre-transfection with $\mathrm{HEa}$ miRNA antagomirs prevented HEamiRNA mimic-induced reduction of VIM, SNAI1, and SNAI2 expression in extravillous trophoblasts (post hoc Tukey's
HSD, $n=10$ samples per group, $P<0.0001$, Fig $6 \mathrm{E}-\mathrm{H}$ ). Thus, our data

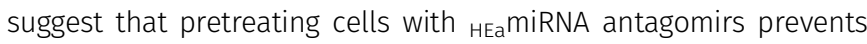
inhibition of EMT pathway members resulting from transfection with HEa miRNA mimics in cytotrophoblasts and extravillous trophoblasts.

\section{HEamiRNAs impair extravillous trophoblast invasion}

Functionally, inhibition of the EMT pathway should reduce trophoblast invasiveness. Thus, we performed a transwell invasion assay using HTR8 extravillous trophoblasts transfected with HEamiRNA mimics and antagomirs. Although ethanol exposure by itself did not impair trophoblast invasion (Fig S6), there was a marginally significant interaction effect between ethanol exposure and HEa miRNA mimic transfection $\left(F_{(1,28)}=3.418, P=0.075\right)$. Thus, a planned 
comparison indicated that transfection with HEamiRNA mimics significantly reduced trophoblast invasion in the context of $320 \mathrm{mg} /$ $\mathrm{dl}$ ethanol co-exposure, relative to the control mimics $(\mathrm{t}(14)=2.762$, $P=0.015)$, consistent with our data demonstrating HeamiRNAs interfere with the EMT pathway (Fig 7A). Contrastingly, transfecting HEamiRNA antagomirs increased invasion in the context of $320 \mathrm{mg} /$ $\mathrm{dl}$ ethanol co-exposure, although this effect was only marginally significant $(t(14)=1.805, P=0.093$, Fig 7B).

HEa MiRNAs retard trophoblast cell cycle progression

Given the proliferative nature of cytotrophoblasts and the intimate relationship between EMT and cell cycle $(64,65)$, we assessed the effects of ethanol and ${ }_{\mathrm{HEa}}$ miRNAs on BeWO cytotrophoblast cell cycle. After pulse-labeling the cells with the nucleic acid analog, EdU, for 1-h, we found that individually transfecting six of the HEamiRNA mimics increased EdU incorporation (unpaired $t$ test, $P<0.05$, false discovery rate [FDR] correction), suggesting an overall increased rate of DNA synthesis (Fig S7A). Contrastingly, simultaneous transfection of ${ }_{\mathrm{HEa}}$ miRNAs significantly reduced EdU incorporation $\left(F_{(1,26)}=59.69, P<0.0001\right)$, mirroring the effects of increasing concentrations of ethanol $\left(R^{2}=0.304, P=0.012\right)$ (Fig S7B and A).

Consistent with the increased rates of DNA synthesis resulting from individual HEamiRNA mimic transfection, individual transfection of HEamiRNAs antagomirs generally reduced EdU incorporation, although only the antagomir to hsa-miR-760 did so

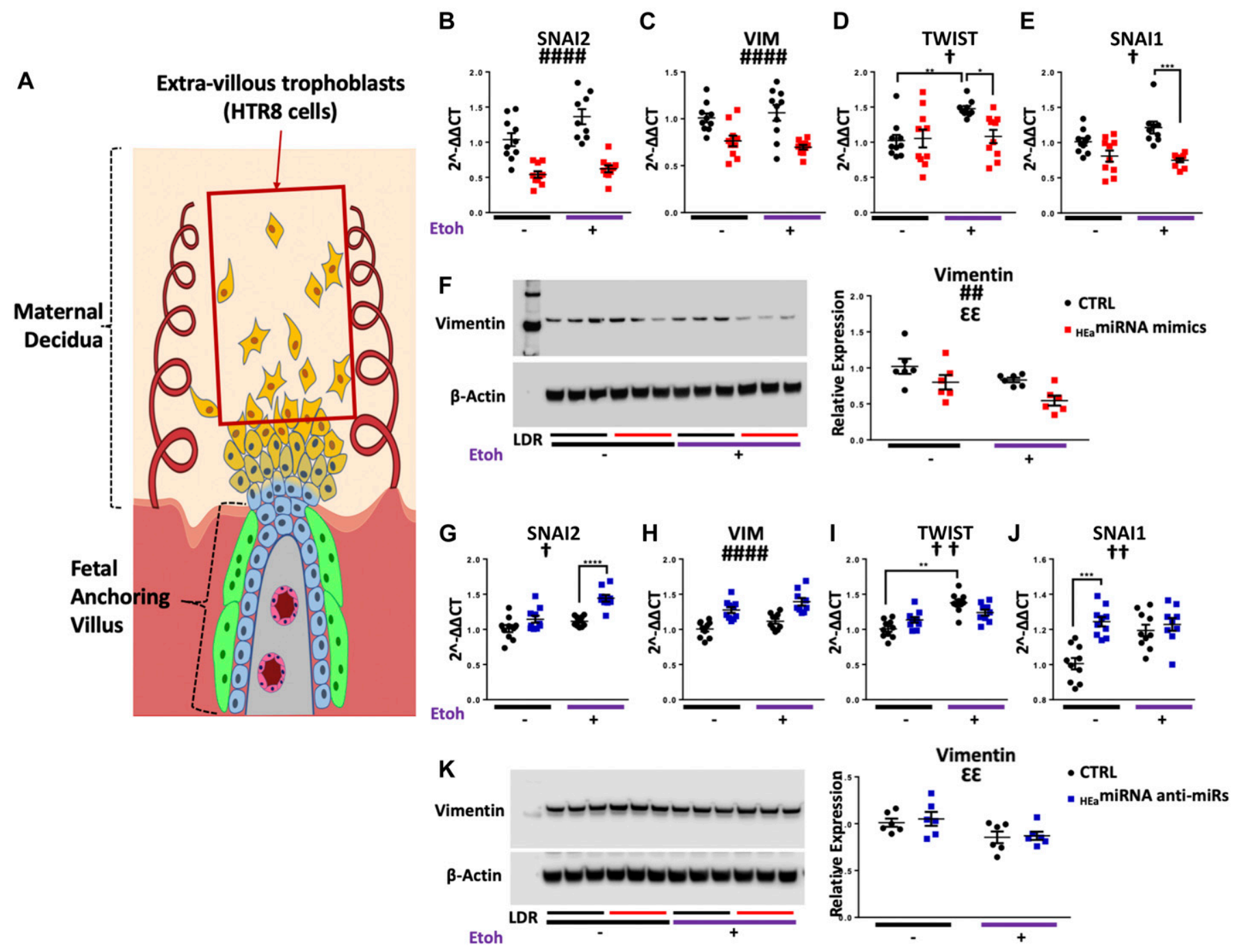

Figure 5. HEamiRNAs interfere with the EMT pathway in HTR8 extravillous trophoblasts.

(A) Diagram of a placental anchoring villous and maternal decidua with the boxed area denoting extravillous trophoblasts. (B-F) Expression of SNAI2 (B), VIM (C), TWIST (D), and SNAl1 transcripts (E) as well as densitometric quantification of vimentin protein levels (F) in HTR8 extravillous trophoblasts following HEamiRNAs or control miRNA overexpression with or without concomitant $320 \mathrm{mg} / \mathrm{dl}$ ethanol exposure. (G-K) Expression of SNAI2 (G), VIM (H), TWIST (I), and SNAl7 transcripts (J) as well as densitometric quantification of vimentin protein levels $(K)$ in HTR8 extravillous trophoblasts following HEamiRNA or control hairpin inhibitor transfection with or without concomitant $320 \mathrm{mg} / \mathrm{dl}$ ethanol exposure. Results are expressed as the mean \pm SEM, LDR = molecular weight ladder, $\mathrm{n}=10$ samples per group; ANOVA: significant main effect of HEamiRNA transfection ( ${ }^{\#} P<0.01$, \#\#\# $P<0.0001$ ), significant main effect of $320 \mathrm{mg} / \mathrm{dl}$ ethanol exposure $\left({ }^{\varepsilon \mathcal{E}} P<0.01\right)$, significant interaction effect $($ HEa $\mathrm{miRNA}$ by $320 \mathrm{mg} / \mathrm{dl}$ ethanol $\left({ }^{\dagger} P<0.05,{ }^{\dagger \dagger} P<0.01\right)$. For post hoc analysis ${ }^{*} P<0.05,{ }^{* \star} P<0.01,{ }^{\star \star *} P<0.001$, and ${ }^{\star \star *} P<0.0001$ by Tukey's HSD. 
A
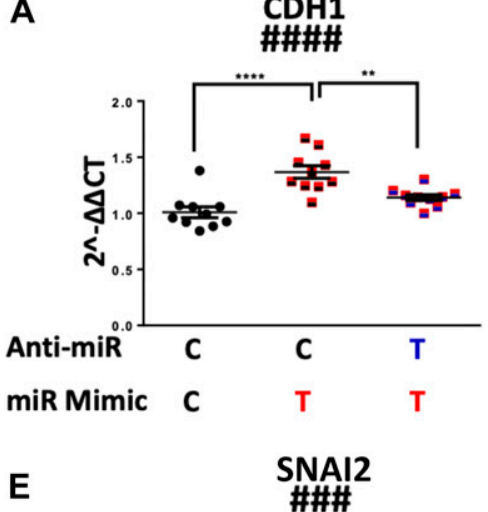

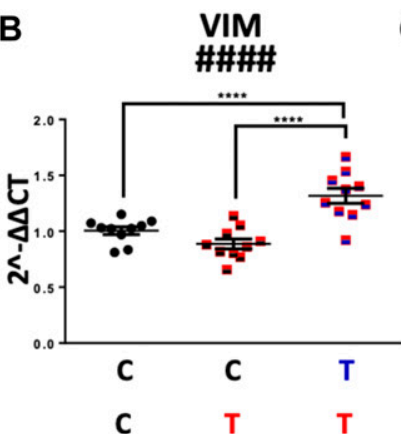

C
C
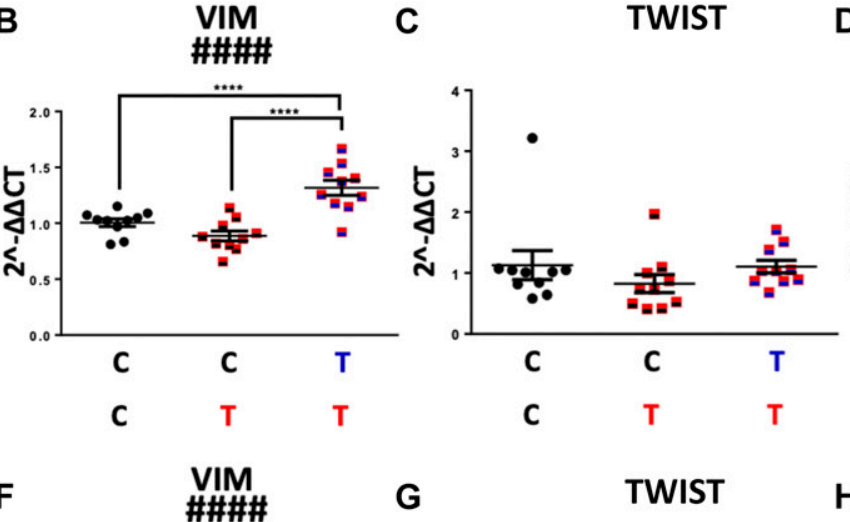

G
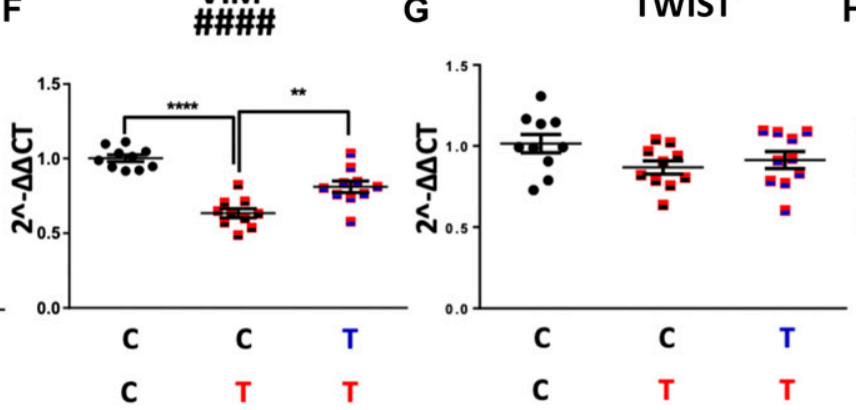

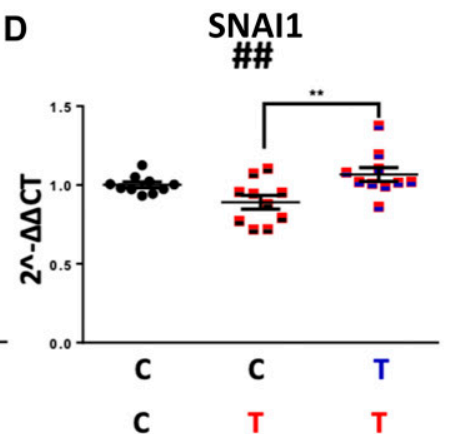

H

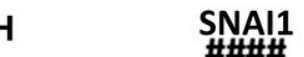

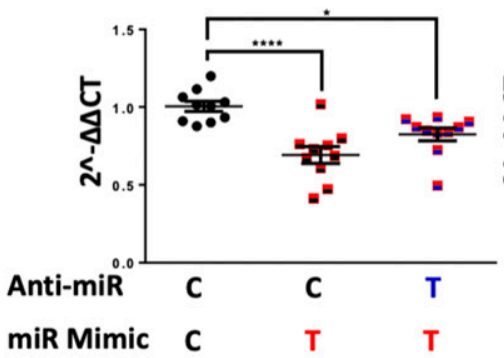
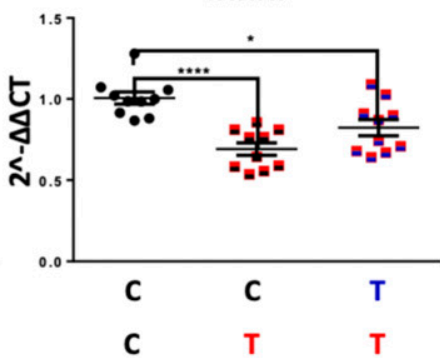

Figure 6. Antagomirs prevent ${ }_{\mathrm{HEa}}$ miRNA-induced impairment of EMT.

(A-D) Expression of CDH1 (A), VIM (B), TWIST (C), and SNAI1 transcripts (D) following control or HEamiRNA hairpin inhibitor transfection followed by control or HEa miRNA overexpression in BeWO cytotrophoblasts. (E-H) Expression of CDH1 (E), VIM (F), TWIST (G), and SNAI1 transcripts (H) following control or HEamiRNA antagomir transfection followed by control or $\mathrm{HEa}_{\mathrm{a}}$ miRNA overexpression in HTR8 extravillous trophoblasts. In subheadings, 'C' denotes control miRNA mimic or hairpin, whereas ' $\mathbf{T}$ ' denotes ${ }_{\mathrm{HEa}}$ miRNA mimic or hairpin inhibitor. Results are expressed as the mean $\pm \mathrm{SEM}, \mathrm{n}=10$ samples per group; ANOVA: significant treatment effect (\#\# $P<0.01$,

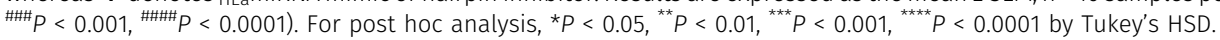

significantly $(\mathrm{t}(110)=3.059, P=0.003$, FDR correction) (Fig S7A). Interestingly, simultaneous administration of antagomirs also reduced EdU incorporation, as observed with the pooled HEamiRNA $\operatorname{mimics}\left(F_{(1,26)}=34.83, P=0.0005\right.$, Fig 8B).

To further characterize the coordinated effect of $\mathrm{HEa}_{\mathrm{miRNAs}}$ on cytotrophoblast cell cycle, we pulse-labeled the cells with EdU for 1-h and, post-fixation, labeled them with 7AAD to segregate cells into three groups: $G_{0} / G_{1}$ (7AADlow, EDU-), $S\left(E D U+\right.$ ), and $G_{2} / M$ (7AADhigh, EDU-). Both $120 \mathrm{mg} / \mathrm{dl}$ and $320 \mathrm{mg} / \mathrm{dl}$ ethanol exposures significantly decreased the proportion of cells in the S-phase, whereas $320 \mathrm{mg} / \mathrm{dl}$ exposure increased the proportion of cells in the $\mathrm{G}_{2} / \mathrm{M}$-phase, consistent with the observed reduction in the rate of DNA synthesis (Fig S7C). Similar to the effects of ethanol exposure, pooled HEamiRNA mimic administration also significantly decreased the proportion of cells in the S-phase $\left(F_{(1,28)}=52.78, P<\right.$ 0.0001 ), whereas increasing the proportion of cells the $G_{2} / M$-phase $\left(F_{(1,28)}=8.395, P=0.007\right)$ and exacerbated alcohol's effects on the cell cycle (Fig 8C). Interestingly, pooled HEamiRNA antagomir administration also reduced the proportion of cells in the S-phase $\left(F_{(1,26)}=14.98, P=0.0007\right)$ and increased the proportion of those in the $G_{2} / M$-phase $\left(F_{(1,26)}=12.38, P=0.002\right)$ (Fig 8D).

As with our EMT gene expression data, pretreatment of cytotrophoblasts with HEamiRNA antagomirs prevented further reduction in the rate of DNA synthesis, or cell cycle retardation, that
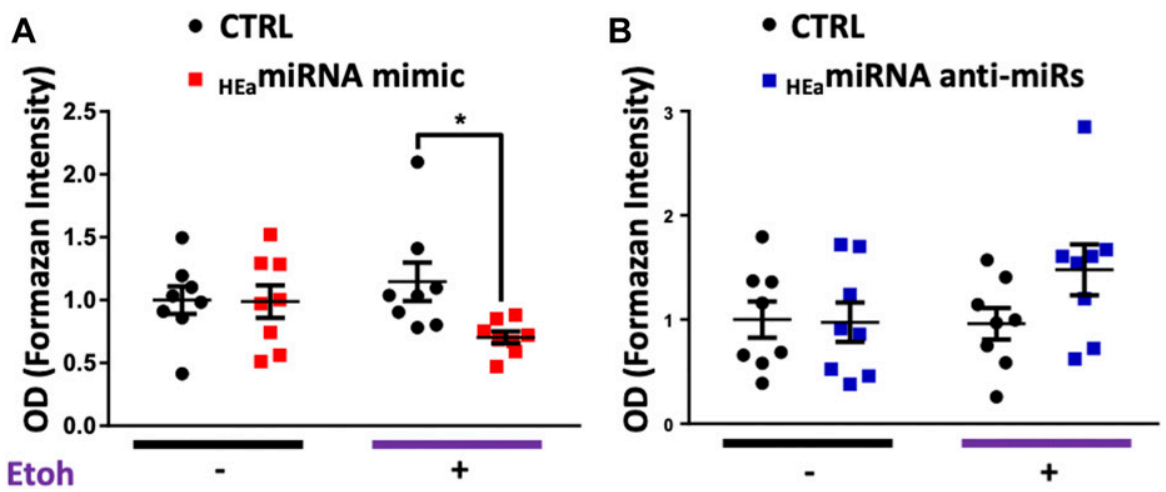

Figure 7. HEamiRNAs impair extravillous trophoblast invasion.

(A, B) Transwell invasion of HTR8 extravillous trophoblasts following transfection with (A) HeamiRNA mimics or (B) hairpin inhibitors with or without concomitant $320 \mathrm{mg} / \mathrm{dl}$ ethanol exposure. $\mathrm{OD}=$ optical density; results are expressed as the mean $\pm S E M ; n=10$ samples per group; $* P<0.05$ by unpaired $t$ test. 
A
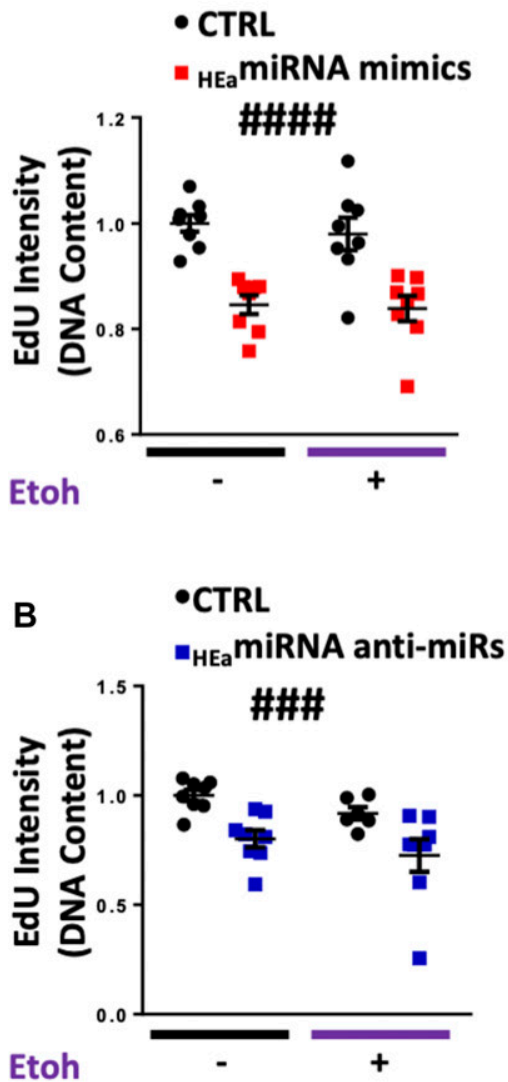

C
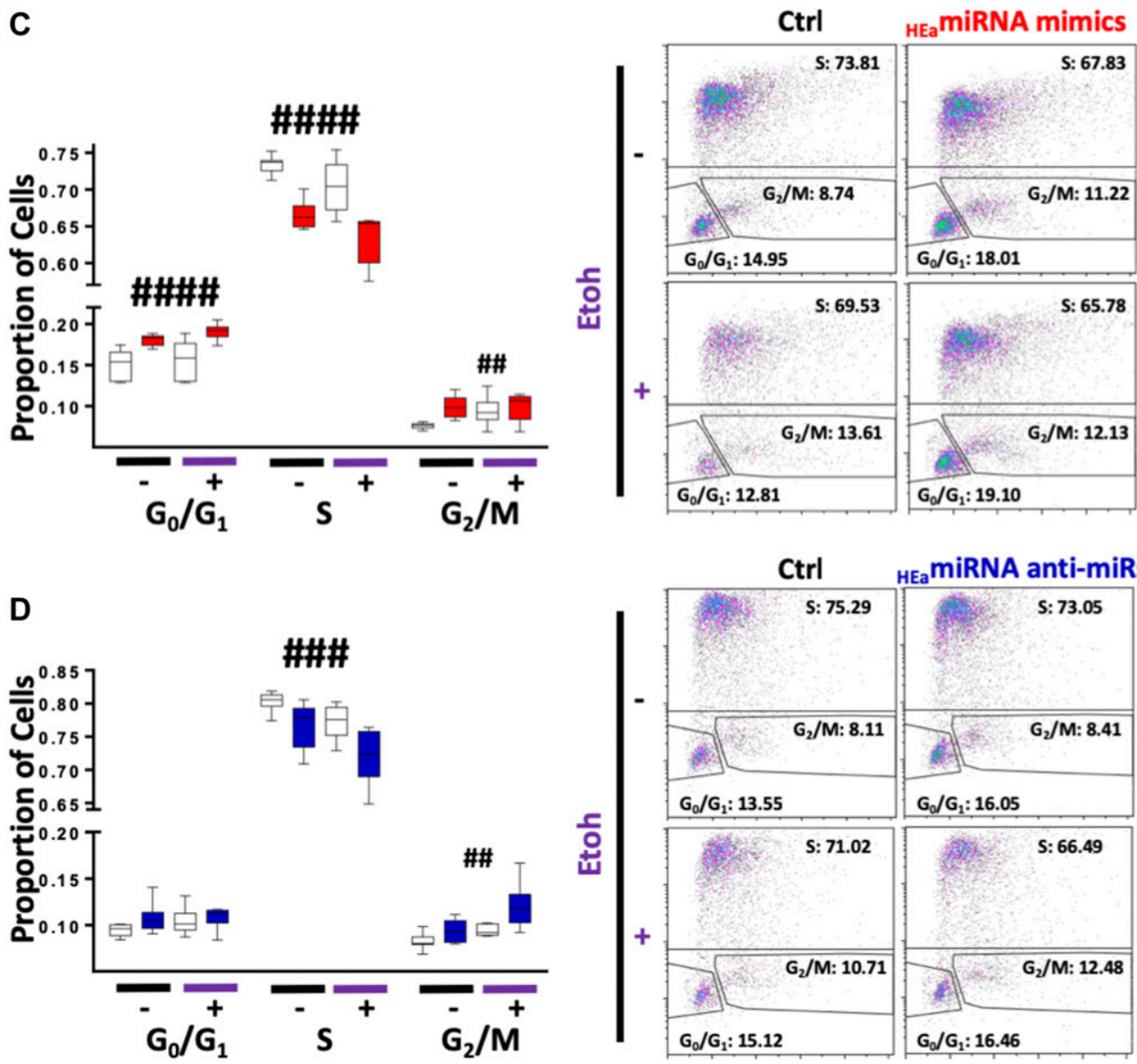

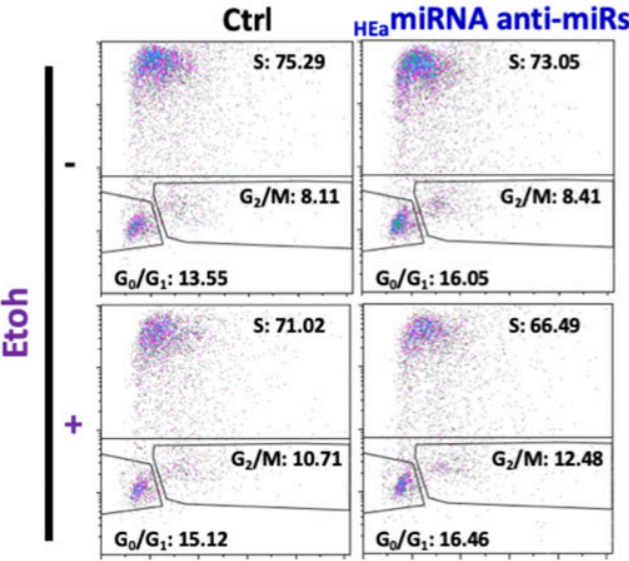

Figure 8. HEa miRNAs cause cell cycle retardation in trophoblasts.

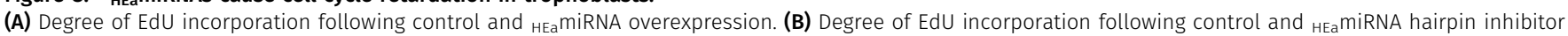
transfection. (C) Box and whisker plot for the proportion of cells in the $\mathrm{G}_{0} / \mathrm{G}_{1}$, $\mathrm{S}$, or $\mathrm{G}_{2} / \mathrm{M}$ phase of the cell cycle following control and $\mathrm{HEa}$ miRNA overexpression. (D) Box and whisker plot for the proportion of cells in the $G_{0} / G_{1}, S$, or $G_{2} / M$ phase of the cell cycle following control and $\mathrm{HEa}$ miRNA hairpin inhibitor transfection with or without concomitant $320 \mathrm{mg} / \mathrm{dl}$ ethanol exposure. For box and whisker plots, bounds of box demarcate limits of the first and third quartile, the line in middle is the median, and whiskers represent the range of data. Representative flow cytometry experiment images are shown on the right. $n=10$ samples per group; ANOVA: significant main effect of

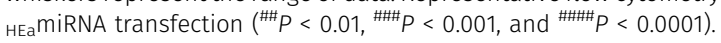

would result from transfection with pooled ${ }_{\mathrm{HEa}}$ miRNA mimics (Fig 9A and B).

HEa miRNAs have minimal effect on cell survival

We next investigated whether ethanol- and HEamiRNA-induced changes in cell cycle were related to an increase in cell death. Only the $320 \mathrm{mg} / \mathrm{dl}$ dose of ethanol exposure demonstrated a slight, but marginally significant effect, of increasing lytic cell death $(t(18)=$ $2.022, P=0.054$ ), although there was no effect on apoptosis (Fig S8A and B). However, the changes in cell cycle following transfection of individual or pooled $\mathrm{HEa}_{\mathrm{m}}$ miRNA mimics were not mirrored by changes in lytic cell death. Nevertheless, two ${ }_{\text {HEa }}$ miRNAs, hsa-miR-671-5p and hsa-miR-449a, did significantly increase apoptosis (unpaired $t$ test, $P<0.05$, FDR correction) (Fig S8C and D).

Contrastingly, transfection of four HEamiRNA antagomirs individually, significantly increased lytic cell death (unpaired t test, all $P<0.05$, FDR correction), with the antagomir to hsa-miR-491-3p also increasing apoptotic cell death $(\mathrm{t}(14)=3.383, P=0.004$, FDR correction, Fig S8C and D). Likewise, transfection of pooled HEamiRNA antagomirs increased lytic cell death $\left(F_{(1,36)}=11.40, P=\right.$ 0.002 ) but did not cause increased apoptosis (Fig S8E-H). Taken together, our data suggest that whereas ethanol exposure may in-

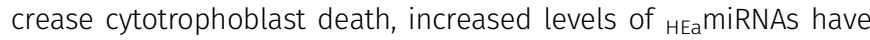
minimal effects on cell death, suggesting that their effect on cell cycle and the EMT pathway is independent of any effect on cell survival.

HEamiRNAs modulate cytotrophoblast differentiation-associated $\mathrm{Ca}^{2+}$ dynamics

HEamiRNAs' effects on EMT pathway member expression, coupled with cell cycle retardation, indicate that ${ }_{\mathrm{HEa}}$ miRNAs influence trophoblast maturation. To model HEamiRNAs' effect on hormoneproducing and calcium-transporting syncytiotrophoblasts (66), we used a well-established protocol of forskolin-induced syncytialization of BewO cytotrophoblasts $(67,68)$. As expected, forskolin treatment induced fusion/syncytialization of cytotrophoblasts resulting in a greater average cell size in the forskolin ${ }^{+}{ }_{\mathrm{HEa}}$ miRNA 

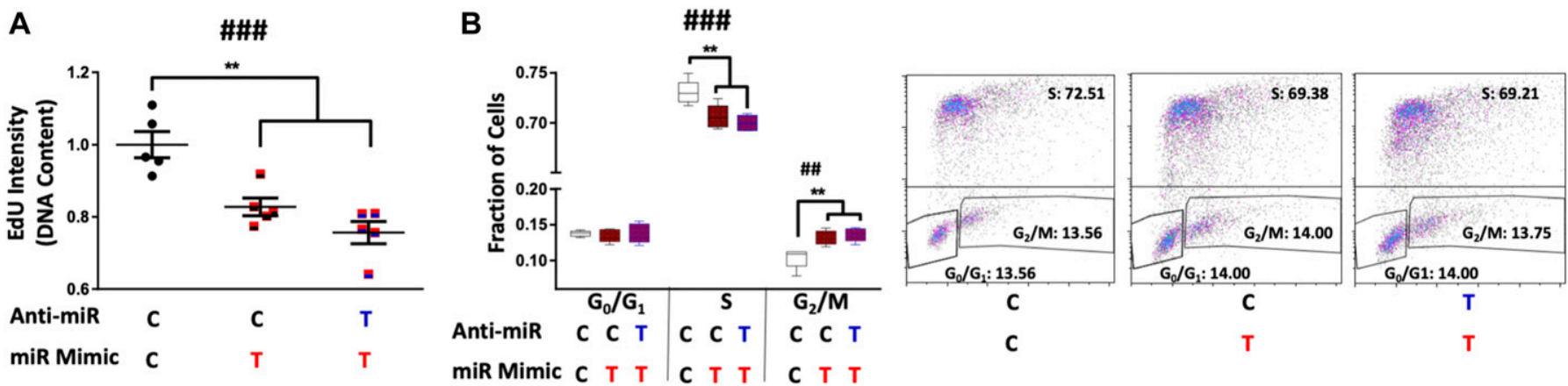

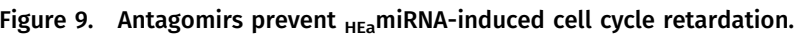

(A) Degree of EdU incorporation following control or $\mathrm{HEa}_{\mathrm{a}} \mathrm{miRNA}$ hairpin inhibitor transfection followed by control or $\mathrm{HEa}_{\mathrm{miRNA}}$ overexpression in BeWO cytotrophoblasts. Results are expressed as the mean $\pm S E M$. (B) Box and whisker plot for the proportion of cells in the $G_{0} / G_{1}, S$, or $G_{2} / M$ phase of the cell cycle following control or HEamiRNA hairpin inhibitor transfection followed by control or HEamiRNA overexpression in BeWO cytotrophoblasts. Bounds of box demarcate limits of the first and third quartile, the line in middle is the median, and whiskers represent the range of data. Representative flow cytometry experiment images are shown on the right. In subheadings, ' $\mathbf{C}$ ' denotes control miRNA mimic or hairpin, whereas ' $T$ ' denotes HEamiRNA mimic or hairpin inhibitor. $\mathrm{n}=5$ samples per group; ANOVA: significant treatment effect $\left({ }^{\# \#} P<0.001\right)$. For post hoc analysis, ${ }^{* *} P<0.01$ by Tukey's HSD.

mimics group $\left(F_{(1,386)}=4.386, P=0.037\right)$. This suggests that the inhibition of EMT by these miRNAs may result in preferential syncytialization instead of differentiation to extravillous trophoblasts (Fig
S9A). Ethanol and forskolin treatment both increased baseline calcium levels, as indicated by the change in fluo-4 fluorescence $\left(F_{(1,426)}=5.593\right.$ and 3.665 , respectively, $P<0.0001$, Figs 10A and S9B-D $)$.

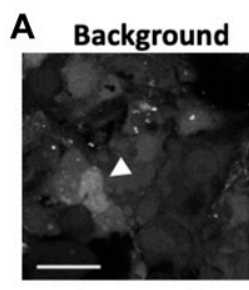

Ionomycin

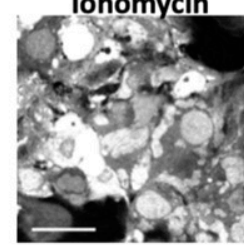

D

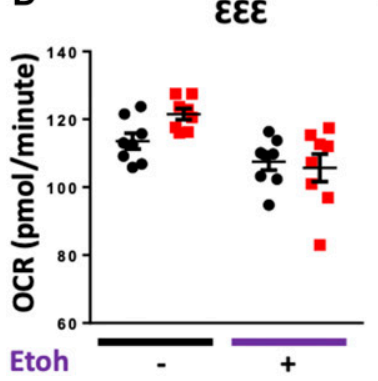

ATP

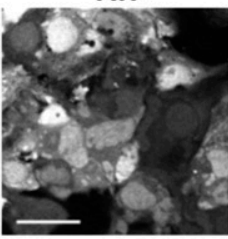

EGTA

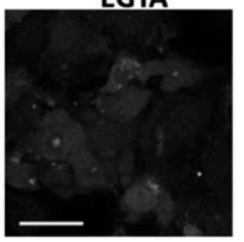

$E$

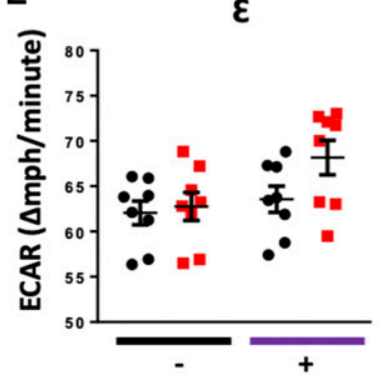

B

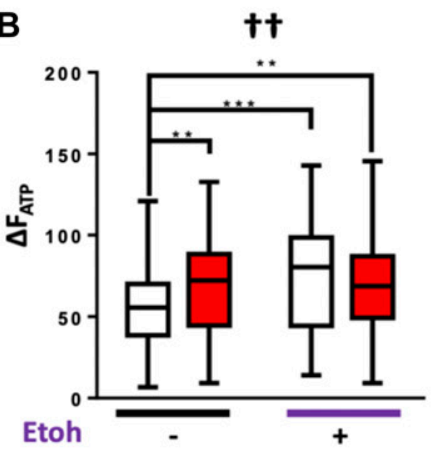

$\mathbf{F}$

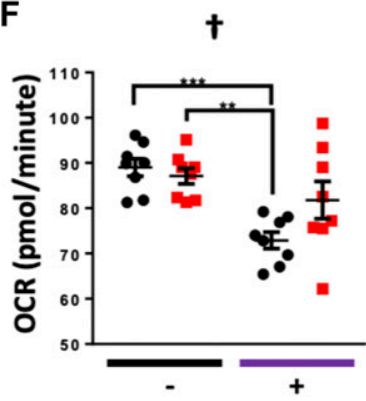

C t+t+
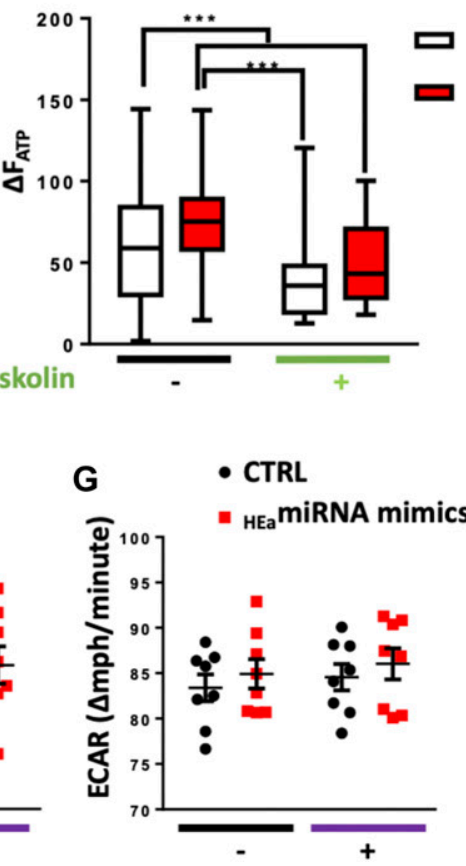

Figure 10. HEamiRNAs modulate differentiation-associated $\mathrm{Ca}^{2+}$ dynamics but have minimal effect on the cellular energetics profile.

(A) Time-lapse confocal images of BeWO cytotrophoblasts loaded with fluo-4 $\mathrm{Ca}^{2+}$ indicator dye under indicated treatment conditions. The arrowhead indicates a fused, multinuclear cell, scale bar (white) is $50 \mu \mathrm{m}$. (B) Box and whisker plot of intracellular calcium levels following acute ATP administration in BewO cytotrophoblasts with control and HEa miRNA overexpression with or without concomitant $320 \mathrm{mg} / \mathrm{dl}$ ethanol exposure. Bounds of box demarcate limits of the first and third quartile, the line in middle is the median, and whiskers represent the range of data. (C) Box and whisker plot of intracellular calcium levels following acute ATP administration in BeWO cytotrophoblasts with control and ${ }_{\mathrm{HEa}}$ miRNA overexpression with or without $20 \mu \mathrm{M}$ forskolin treatment. (D-G) Baseline OCR (D), baseline ECAR (E), stressed OCR (F), and stressed ECAR (G) in BeWO cytotrophoblasts with control and HEamiRNA overexpression with or without concomitant 320 mg/dl ethanol exposure. Metabolic stress was induced by treatment with $1 \mu \mathrm{M}$ oligomycin and $0.125 \mu \mathrm{M}$ (FCCP). Results are expressed as the mean \pm SEM. $\mathrm{n}=10$ samples per group; ANOVA: significant main effect of $320 \mathrm{mg} / \mathrm{dl}$ ethanol exposure $\left({ }^{\varepsilon} P<0.05,{ }^{\varepsilon \varepsilon \varepsilon} P<0.001\right.$ ), significant interaction effect ( ${ }_{\text {HEa }}$ miRNA by $320 \mathrm{mg} / \mathrm{dl}$ ethanol $\left[{ }^{\dagger} P<0.05\right.$, ${ }^{+\dagger} P<0.01$, and $\left.\left.{ }^{+++\dagger} P<0.0001\right]\right)$. For post hoc analysis, ${ }^{\star} P<0.05,{ }^{\star \star} P<0.01,{ }^{\star \star \star} P<0.001$, and ${ }^{\star \star \star} P<0.0001$ by Tukey's HSD. 
The effect of ethanol on baseline calcium was abrogated by HEamiRNAs, whereas heamiRNAs + forskolin was not significantly different to forskolin alone, indicating that forskolin

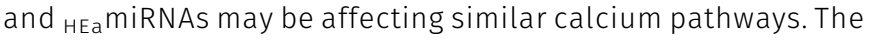
conversion of cytrophoblasts to syncytiotrophoblasts is accompanied by an increase in endoplasmic reticulum, which could increase calcium-buffering capabilities in response to ethanol stress on the cells; thus, HEamiRNA-induced syncytialization pathways may be protective against ethanol stress.

Adaptations to cellular stress can also be seen in alterations to cellular energetics in response to ethanol, as ethanol-exposed BeWO cells showed decreased baseline and stressed oxygen consumption rates $(\mathrm{OCR}) \mathrm{F}_{(1,28)}=15.55$ and 16.91, $P=0.0005$ and 0.0003 , respectively) and increased extracellular acidification rates
(ECAR) $\left(F_{(1,28)}=4.868, P=0.036\right)$. However, HEa miRNAs had minimal effects on metabolic activity (Fig 10D-G).

Extracellular ATP has been shown to inhibit trophoblast migration (69) and can directly stimulate increased intracellular calcium elevations through purinergic receptors ubiquitously present on trophoblasts (70). Both HEamiRNA and ethanol administration significantly increased intracellular calcium in response to acute ATP administration $\left(F_{(1,426)}=10.34\right.$ and $F_{(1,386)}=16.30, P=0.001$ and $P<$ 0.0001 , respectively) (Fig 10B). This may be indicative of a lack of down-regulation of purinergic receptors required in trophoblast migration as part of the interrupted EMT pathway. Forskolin-induced maturation decreased calcium response to ATP $\left(F_{(1,386)}=50.72, P<\right.$

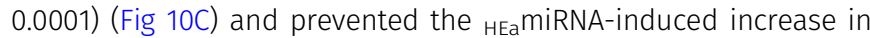
ATP response. These data agree with previous studies showing
A

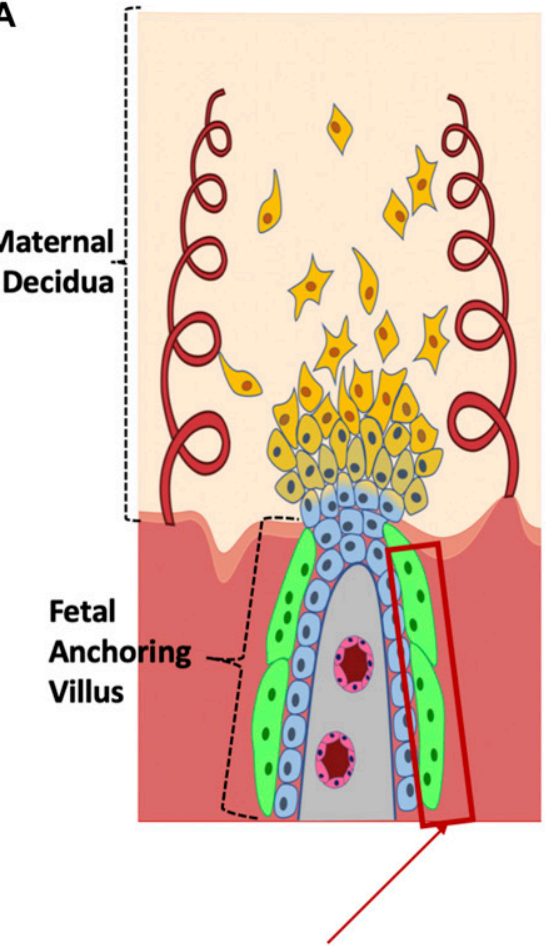

Syncytiotrophoblasts (Forskolin-treated BeWO cells)
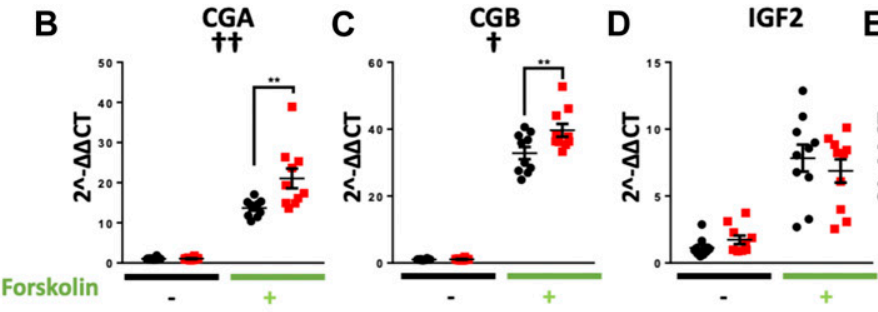

E
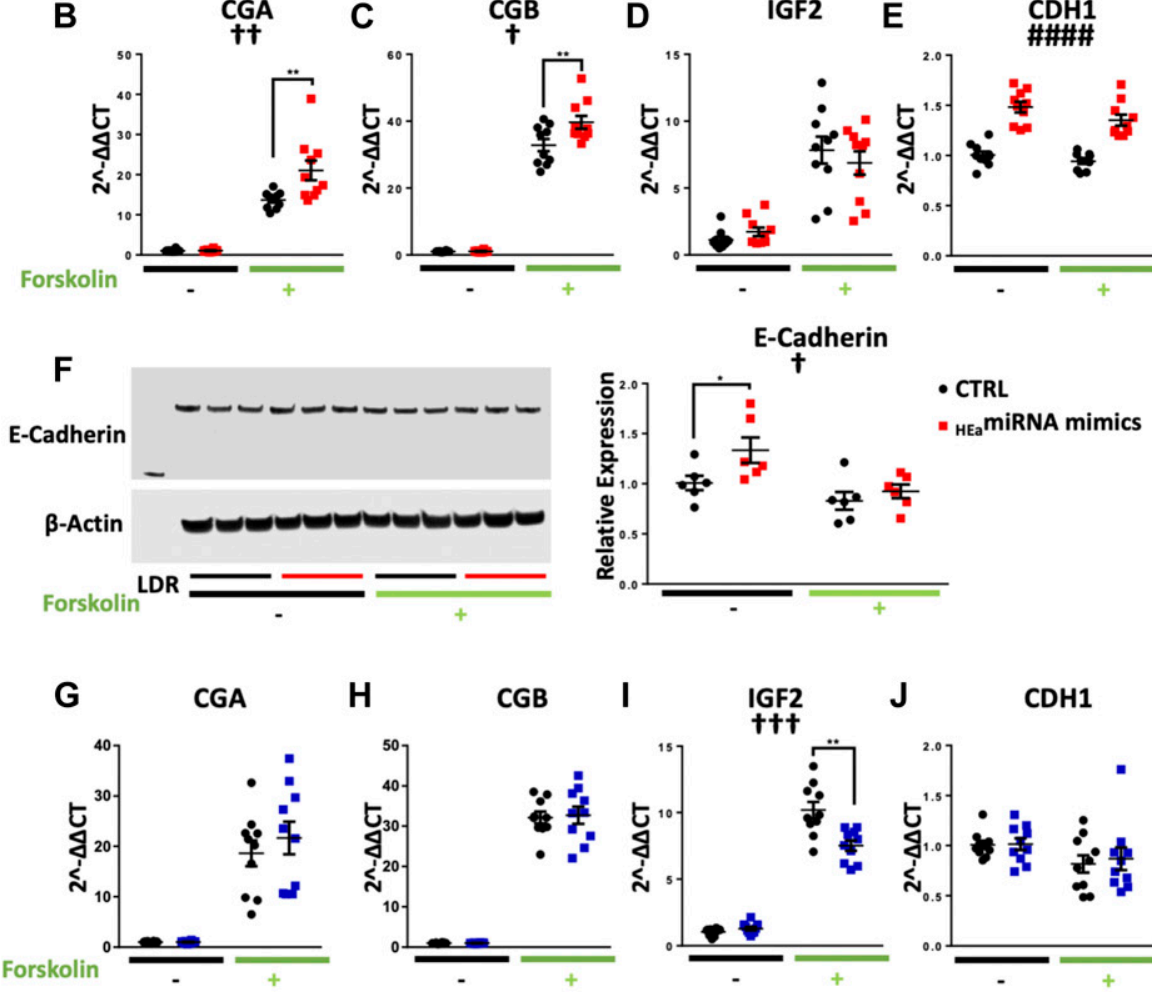

$\mathbf{J}$

CDH1

K

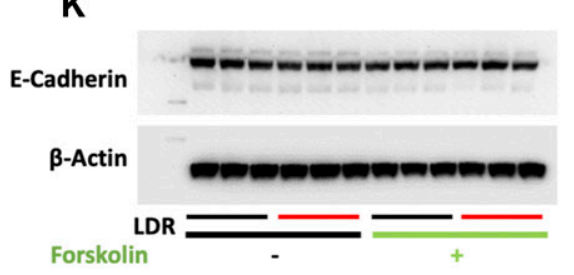

E-Cadherin

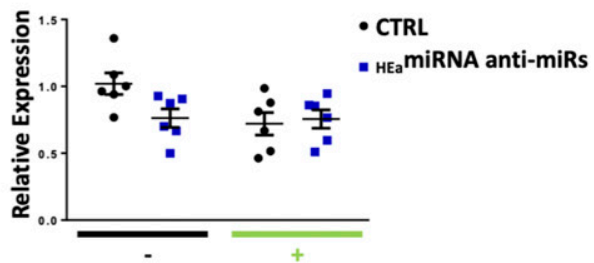

Figure 11. HЕamiRNAs promote syncytialization dependent hCG production.

(A) Diagram of a placental anchoring villous and maternal decidua with the boxed area denoting syncytiotrophoblasts. (B-F) Expression of CGA (B), CGB (C), IGF2 (D), and CDH1 transcripts (E) and densitometric quantification of E-Cadherin protein levels (F) in BeWO cytotrophoblasts following $\mathrm{HEa}$ miRNAs or control miRNA overexpression with or without $20 \mu \mathrm{M}$ forskolin treatment. (G-K) Expression of CGA (G), CGB (H), IGF2 (I), and CDH1 transcripts (J) and densitometric quantification of E-Cadherin

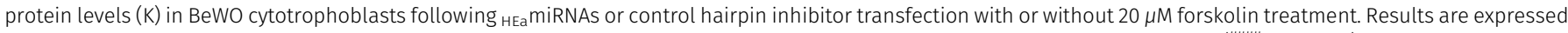
as the mean $\pm S E M, L D R=$ molecular weight ladder, $n=10$ samples per group; ANOVA: significant main effect of ${ }_{\text {HEa }}$ miRNA transfection $\left({ }^{\# \# \# ~} P<0.0001\right)$, significant interaction

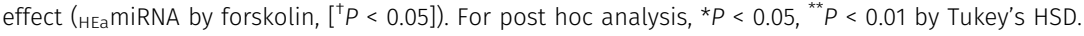


increased nuclear trafficking of ionotropic receptor P2X7 and more localized P2X4 expression over placental development, which may decrease the overall calcium influx in response to ATP (71).

\section{HEamiRNAs promote syncytialization-dependent hormone production}

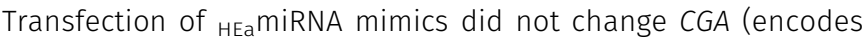
Chorionic gonatropin alpha), CGB (encodes Chorionic gonadoptropin beta), or IGF2 (encodes Insulin-like growth factor 2) transcript expression relative to the control in non-syncytialized trophoblasts. However, following forskolin-induced syncytialization of BeWO cytotrophoblasts (Fig 11A), HeamiRNA mimics significantly increased expression of CGA and CGB (post hoc Tukey's HSD, $n=10$ samples per group, $P=0.001$ and 0.005 , respectively). Consistent with our previous results, HEamiRNA mimics also increased $\mathrm{CDH} 1$ expression in both cytotrophoblasts and syncytiotrophoblasts $\left(F_{(1,20)}=5.286, P=0.032\right)$; there was also a main effect of syncytialization on $\mathrm{CDH} 1$ expression, as has been previously reported $\left(F_{(1,36)}=3.391, P=0.034\right.$, Fig 11B-E). Likewise, HEamiRNAs increased $E$-Cadherin protein expression $\left(F_{(1,20)}=5.286\right.$, $P=0.032)$, whereas forskolin decreased it $\left(F_{(1,20)}=10.24, P=0.005\right)$ (Fig 11F). On the other hand, there was no effect of HEa miRNA antagomirs on CGA and CGB expression, although we did observe a decrease in IGF2 transcript expression, following syncytialization, relative to controls (post hoc Tukey's HSD, $n=10$ samples per group, $P=0.001$ ) (Fig 11G-J).

Given that HEamiRNAs promote syncytialization-dependent hormone production, we next investigated maternal plasma levels of intact hCG in our Ukraine birth cohort. Plasma hCG levels were nonsignificantly increased in the second trimester of $\mathrm{HEa}$ group mothers relative to their UE counterparts, consistent with previous studies (72). During the third trimester, however, hCG levels remained significantly elevated in $\mathrm{HEa}$ group mothers compared with the UE group (median test, $\mathrm{n}=23$ samples in $\mathrm{HEa}$

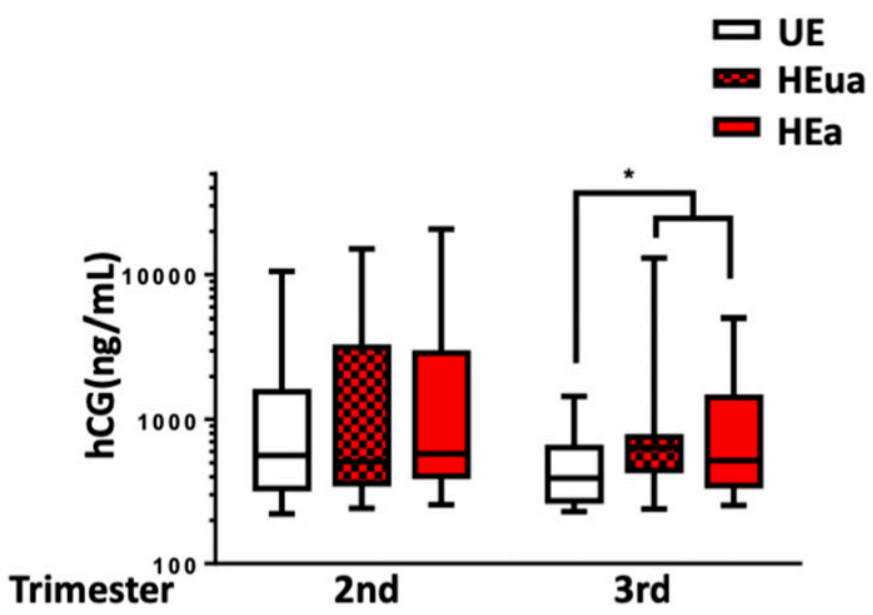

Figure 12. PAE elevates third trimester maternal hCG.

Box and whisker plot of the second and third trimester maternal hCG levels in UE, HEua, and HEa group mothers of our Ukrainian birth cohort. Bounds of box demarcate limits of the first and third quartile, the line in middle is the median, and whiskers represent the range of data. Results are expressed as the mean \pm SEM, $n=22-23$ samples per group; ${ }^{*} P=0.03$ (Mood's median test, $X^{2}=7.043, \mathrm{df}=2$ ). group and $\mathrm{n}=22$ for HEua and UE groups, $P=0.03$ ) (Fig 12). Furthermore, there was no significant difference of gestational age at blood draw between the different groups indicating the increased level of hCG in the HEa group was not confounded by gestational age at which blood was sampled (Fig S10) (73). Interestingly, both alcohol and hCG levels were negatively associated with gestational age at delivery (GAD), with a significant interaction between periconceptional alcohol exposure and hCG levels on GAD (Table S2). Taken together, our data suggest HEamiRNAs may contribute to PAE-dependent increases in hCG levels during pregnancy.

\section{HEa miRNAs reduce fetal growth}

To investigate the functional consequences of elevated circulating HEamiRNA levels, we administered miRNA mimics for the eightmouse homolog ${ }_{\mathrm{HEa}} \mathrm{miRNAs}$, or a negative control mimic, through tail vein injection to pregnant mouse dams on GD10. On GD18, growth parameters of male and female fetuses were assessed separately, and data from all same-sex fetuses from a single pregnancy were averaged into one data point. Dams-administered HEa miRNA mimics produced smaller fetuses than those administered control mimics, according to all collected measures of fetal size: fetal weight $\left(F_{(1,17)}=9.92, P=0.006\right)$, crown-rump length $\left(F_{(1,17)}=\right.$ 9.89, $P=0.006)$, snout-occipital distance $\left(F_{(1,17)}=9.09, P=0.008\right)$, and biparietal diameter $\left(F_{(1,17)}=5.99, P=0.026\right)$ (Fig 13B-E). Interestingly, placental weights were also significantly reduced in mice treated

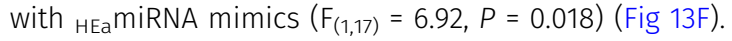

Following tail vein administration of two human-specific sentinel miRNAs, miR-518f-3p and miR-519a-3p, we found a high biodistribution of both miRNAs in the placenta, comparable with levels seen in the liver and spleen (Fig S11A and B). Thus, to determine whether HEamiRNA's effects on fetal growth could result from their actions on the placenta, we quantified the placental expression of

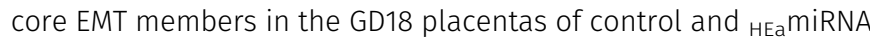
fetuses. HEamiRNA administration significantly reduced expression of mesenchymal-associated transcript VIM $\left(F_{(1,14)}=14.23, P=0.002\right)$ and SNAI2 $\left(F_{(1,14)}=5.99, P=0.028\right)$ with a significant sex by HEa $_{\text {miRNA }}$ interaction effect on $\operatorname{SNAl1}\left(F_{(1,66)}=5.55, P=0.034\right)$ and $\operatorname{CDH1}\left(F_{(1,14)}=\right.$ $6.01, P=0.028)$ (Fig 14A-E). Interestingly, and in line with our in vitro

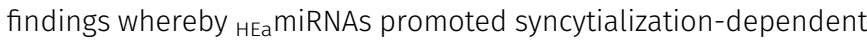
cell fusion and hCG production, HEamiRNA administration significantly increased expression of the mRNA transcript for SynB, a gene that is important for syncytiotrophoblast maturation $\left(F_{(1,66)}=4.11\right.$, $P=0.047$ ) (Fig 14F).

\section{Discussion}

We previously reported that gestational elevation of 11 maternal plasma miRNAs predicted which PAE infants would exhibit adverse

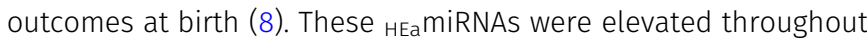
mid and late-pregnancy, encompassing critical periods for fetal development, and were predicted to target the EMT pathway (8). In this study, we tested this prediction by adopting rodent and macaque gestational moderate alcohol self-administration paradigms. Despite differences in their placental anatomy $(74,75,76,77)$, 
A

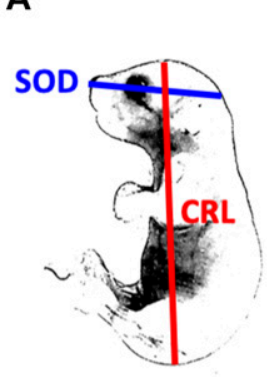

D

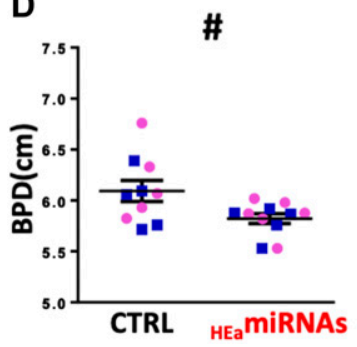

BPD

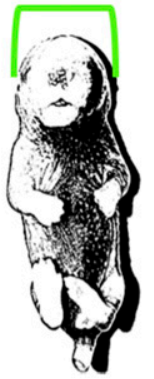

E
B

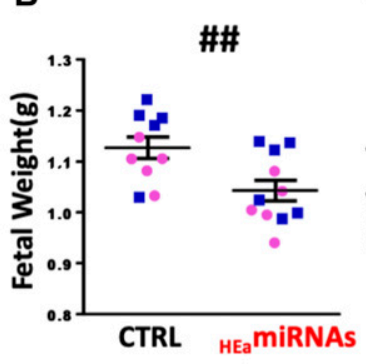

C

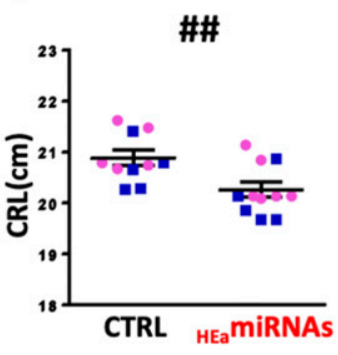

Figure 13. HЕamiRnAs restrict fetal growth.

(A) Schematic for measures of crown rump length (CRL), biparietal diameter (BPD), and snout-occipital distance (SOD). (B-F) Fetal weight (B), crown-rump length (C), biparietal diameter (D), snout-occipital distance (E), and placental weight (F) at GD18 following administration of

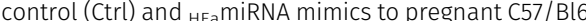
dams on GD10. Dots represent median measures of fetal size and placental weights from male and female offspring in independent litters. There were no significant differences in litter sizes (Ctrl: 8.2 and HEamiRNAs: 8.5) or sex ratios (Ctrl: 0.86 and HeamiRNAs: 1.21) between treatment conditions $(P>0.5$ for all measures). Results are expressed as the mean $\pm \mathrm{SEM}$, $n=5-6$ separate litters per treatment condition; ANOVA: significant main effect of ${ }_{\text {HEa }}$ miRNA administration $\left({ }^{\#} P<\right.$ 0.05 and $\left.{ }^{\#} P<0.01\right)$

we are the first to report that PAE impairs placental EMT across species, indicating a conserved effect of PAE on placental development. In addition, we found that ${ }_{\text {HEa }}$ miRNAs collectively, but not individually, mediated the effects of PAE on core EMT pathway members and that, together, they inhibited EMT in human trophoblast culture models. Although we assessed the effects of HEamiRNAs on core EMT components $(10,14,15,59,60,61,62)$, analysis of their 3' UTRs indicates that these are unlikely to be the direct targets of HEa miRNA action. Additional studies will be needed

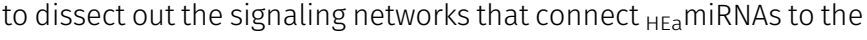
assessed EMT components.

Interestingly, HeamiRNAs also promoted syncytialization (forskolin)dependent hCG expression, mirroring the elevation of third trimester maternal hCG levels in the PAE group within our clinical cohort. This late-gestation elevation of hCG levels may serve as a compensatory mechanism to prevent the preterm birth associated with PAE, as hCG during late gestation is hypothesized to promote uterine myometrial quiescence $(78,79)$. In support of this hypothesis, we found significant negative associations between both hCG levels and alcohol consumption with GAD. Furthermore, there was a significant interaction between periconceptional alcohol exposure and hCG levels, with higher hCG levels corresponding to a smaller effect of alcohol exposure at conception on GAD, indicating that hCG moderates the effect of alcohol on age at delivery (Table S2).

Since HEamiRNAs collectively prevented trophoblast EMT, we hypothesized that, as a functional consequence, these maternal
A
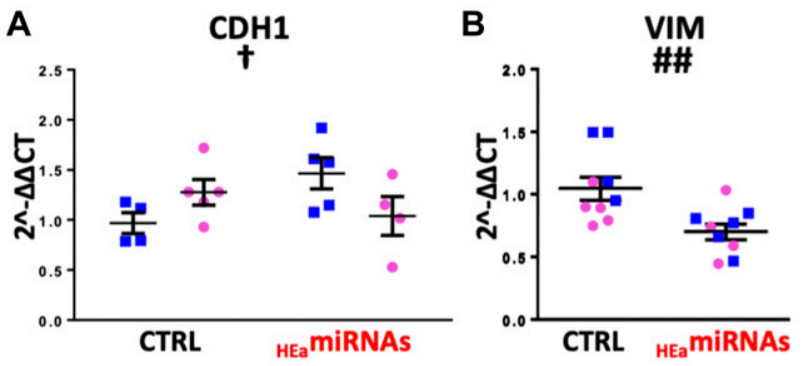

C
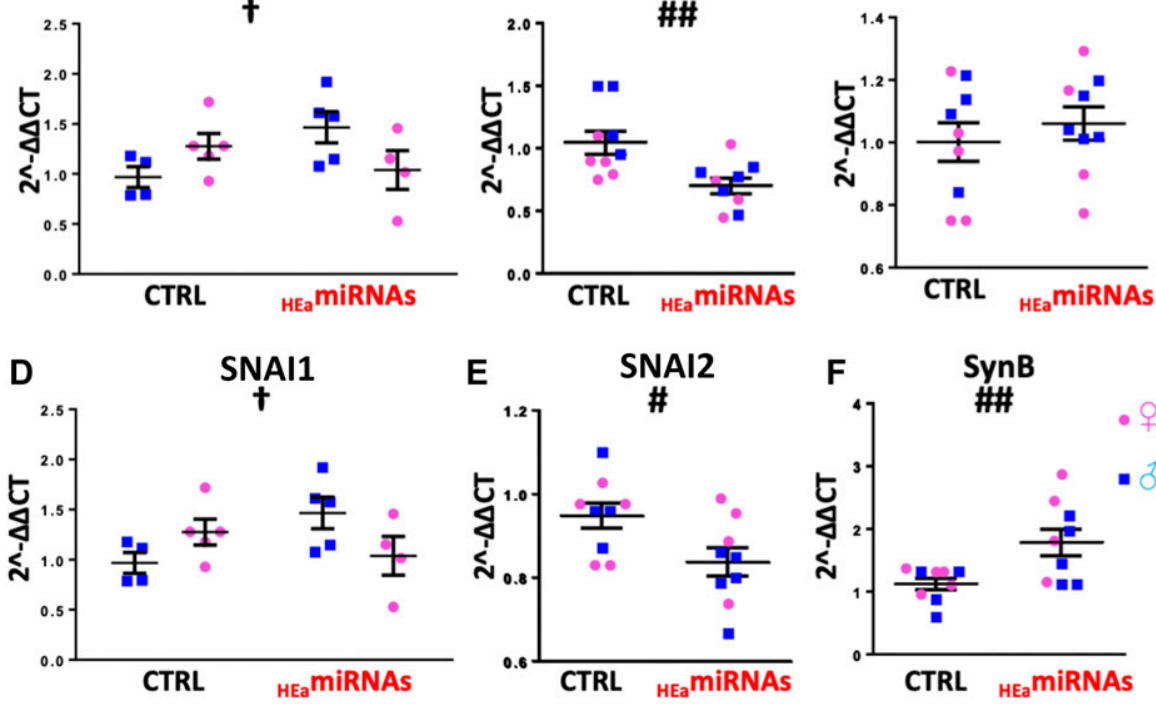

E

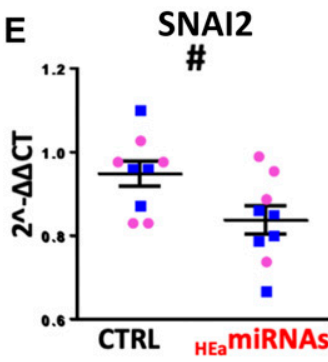

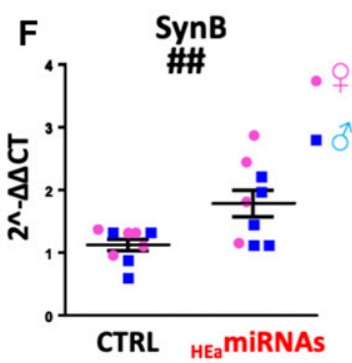

Figure 14. HEamiRNAs interfere with EMT in the placenta.

(A-F) Expression of CDH1 (A), VIM (B), TWIST (C), SNAI1 (D), and SNAI2 (E) and SynB transcripts (F) in GD18 placenta following administration of control (Ctrl) and HEa miRNA mimics to pregnant C57/Bl6 dams on GD10. Dots represent median expression values of male and female offspring in independent litters. Results are expressed as the mean $\pm \mathrm{SEM}, \mathrm{n}=5-6$ separate litters per treatment condition, ANOVA: significant main effect of ${ }_{\text {HEa }}$ miRNA administration $\left({ }^{\#} P<0.05,{ }^{\# \#} P<0.01\right)$, significant interaction effect (fetal sex by неamiRNA administration, $\left.\left[{ }^{\dagger} P<0.05\right]\right)$. For post hoc analysis, ${ }^{*} P<$ 0.05 by Tukey's HSD. 
miRNAs would also inhibit fetal growth. When we delivered 8 of the 11 HEa miRNAs known to be present in mouse, to pregnant dams during the period of placental branching morphogenesis and endometrial invasion, and when EMT is particularly active, we found that $\mathrm{HEa}_{\mathrm{m}}$ miRAs reduced fetal growth. Importantly, ethanol exposure during this period has also been shown to result in fetal growth deficits and dysmorphia in rodent PAE models $(80,81)$, suggesting that maternal miRNA-mediated deficits in trophoblast invasion may mediate some of the effects of PAE on fetal growth. In support of this, we found placentas from the HEamiRNA-treated group had impaired expression of core EMT pathway members. This disruption of placental EMT may also have implications for placental vascular dynamics, as we have also previously observed in mouse models (82). The nonhuman primate tissue analyzed here was also derived from animals that were characterized in vivo using MRI and ultrasound imaging, which demonstrated that maternal blood supply to the placenta was lower in ethanol-exposed animals compared with controls and that oxygen availability to the fetal vasculature was reduced (83).

HEa miRNAs may mediate other pregnancy-associated pathologies, aside from PAE. We identified numerous studies that reported increased circulating and placental levels of at least 8 of 11 HEamiRNAs in gestational pathologies arising from placental dysfunction. For example, elevated levels of one HEa miRNA, miR-519a-3p, a member of the placentally expressed C19MC family cluster, was reported in the placentae of patients with preeclampsia, recurrent spontaneous abortion, and intrauterine growth restriction $(29,30,45$, 46). Interestingly, collective overexpression of the 59 C19MC miRNAs inhibits trophoblast migration, explaining their enrichment in the non-migratory villous trophoblasts and suggests their downregulation is necessary for maturation into invasive extravillous trophoblasts (84). Thus, a greater understanding of the placental

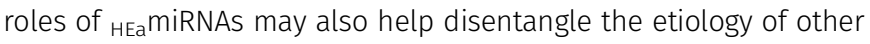
pregnancy complications. We also observed that overexpression of more restricted subsets of ${ }_{\mathrm{HEa}}$ miRNAs associated with preeclampsia, fetal growth restriction, and spontaneous abortion or preterm labor also partly promoted EMT transcript signatures, contrasting with the

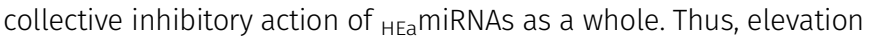
of some subsets of HEamiRNAs may constitute a compensatory mechanism aimed at minimizing placental pathologies, although their potential protective effects are masked by the collective elevation of HEamiRNAs.

Although we did not investigate the effects of PAE on EMT in nonplacental organs, it is likely that PAE broadly disrupts EMT in multiple fetal compartments. Developmental ethanol exposure has been shown to inhibit the EMT-dependent migration of neural crest progenitors involved in craniofacial development, explaining the facial dysmorphology seen in fetal alcohol syndrome and FASDs $(85,86)$. Outside of its effects on the neural crest, PAE is significantly associated with various congenital heart defects, including both septal defects and valvular malformations $(87,88,89,90)$. Given that development of heart depends on EMT within the endocardial cushions $(91,92)$, disruption of endocardial EMT could explain both the valvular and septal malformation associated with PAE.

Collectively, our data on HEamiRNAs suggest miRNA-based interventions could minimize or reverse developmental effects of PAE and other placental-related pathologies. miRNA-based therapeutic approaches have been advanced for other disease conditions (93, 94). However, our data also suggest the effects of combinations of miRNAs are not a sum of their individual effects. Functional synergy between clusters of co-regulated miRNAs may be a common feature in development and disease. For instance, in 2007, we presented early evidence that ethanol exposure reduced miR-335, -21, and -153 in neural progenitors and that coordinate reduction in these miRNAs yielded net resistance to apoptosis following ethanol exposure (95). In that study, we also showed that coordinate knockdown of these three miRNAs was required to induce mRNA for Jagged-1, a ligand for the Notch cell signaling pathway, an outcome that was not recapitulated by knocking down each miRNA individually (95). More recently, combined administration of miR-21 and miR-146a has been shown to be more effective in preserving cardiac function following myocardial infarction than administration of either of these miRNAs alone (96). Although miRNA synergy has not been explored in detail, these data show that new biology may emerge with admixtures of miRNAs and that therapeutic interventions may require the use of such miRNA admixtures rather than single miRNA molecules, as have been used in clinical studies to date.

In conclusion, we have observed that a set of 11 miRNAs, predictive of adverse infant outcomes following PAE, collectively mediate the effects of alcohol on the placenta. Specifically, elevated levels of these miRNAs together, but not individually, promote an aberrant maturational phenotype in trophoblasts by inhibiting core members of the EMT pathway and promoting cell stress and syncytialization-dependent hormone production. Although extensive research has established circulating miRNAs as biomarkers of disease, our study is one of the first to show how these miRNAs explain and control the disease process themselves. Functionally, we find that these miRNAs are clinically correlated with measures of fetal development and directly cause intrauterine growth restriction when administered in vivo. Our work suggests that a greater understanding for the role of ${ }_{\mathrm{HEa}}$ miRNAs during development, and their role in coordinating the EMT pathway in the placenta and other developing tissues, will benefit the understanding of FASDs and other gestational pathologies and potentially lead to effective avenues for intervention.

\section{Materials and Methods}

\section{Mouse model of PAE}

C57/BL6J mice (The Jackson Laboratory) were housed under reverse 12-h dark/12-h light cycle (lights off at 08:00 h). PAE was performed using a previously described limited access paradigm of maternal drinking $(97,98)$. Briefly, 60-d-old female mice were subjected to a ramp-up period with $0.066 \%$ saccharin containing $0 \%$ ethanol $(2 \mathrm{~d})$, $5 \%$ ethanol ( $2 \mathrm{~d}$ ), and finally $10 \%$ ethanol for 4-h daily from 10:00 to 14:00 beginning 2 wk before pregnancy, continuing through gestation (Fig S2A). Female mice offered $0.066 \%$ saccharin without ethanol during the same time period throughout pregnancy served as controls. Tissue from the labyrinth, junctional, and decidual zone of male and female gestational day 14 (GD14) placentae were 
microdissected, snap-frozen in liquid nitrogen, and stored at $-80^{\circ} \mathrm{C}$ preceding RNA and protein isolation.

\section{Mouse model for ${ }_{\text {HEa }}$ miRNA overexpression}

For systemic administration of miRNAs, previously nulliparous C57/BL6NHsd dams (Envigo) were tail vein-injected on GD10 with either $50 \mu \mathrm{g}$ of miRNA miRVana mimic negative control (Cat No. 4464061; Thermo Fisher Scientific) or pooled HEa miRNA miRVana mimics in In-vivo RNA-LANCEr II (3410-01; Bioo Scientific), according to the manufacturer's instructions. The $50 \mu \mathrm{g}$ of pooled $\mathrm{HEa}_{\mathrm{a}} \mathrm{miRNA}$ mimics consisted of equimolar quantities of mmu-miR-222-5p, mmu-miR-187-5p, mmu-miR-299a, mmu-miR-491-3p, miR-760-3p, mmu-miR-671-3p, mmu-miR-449a-5p, and mmu-miR-204-5p mimics. For biodistribution studies, $50 \mu \mathrm{g}$ of pooled equimolar quantities of hsa-miR-519a-3p and hsa-miR-518f-3p mimics were injected via tail vein. These human miRNAs were selected because no mouse homologs are known to exist and consequently, estimates for organ distribution of exogenous miRNAs in the mouse are unlikely to be contaminated by the expression of endogenous murine miRNAs. GD10 is a time point near the beginning of the developmental period of branching morphogenesis, immediately following chorioallantoic attachment, during which the placenta invades the maternal endometrium (99). At GD18, pregnancies were terminated with subsequent quantification of fetal weight, crown-rump length, snout-occipital distance, biparietal diameter, and placental weight (Fig 13A). Subsequently, tissue was snapfrozen in liquid nitrogen and stored at $-80^{\circ} \mathrm{C}$ preceding RNA isolation.

\section{Rat model of PAE}

Outbred nulliparous Sprague Dawley rats were housed under a 12-h light/12-hour dark cycle. PAE in Sprague Dawley was conducted according to our previously published exposure paradigm $(20,100)$. Briefly, dams were given a liquid diet containing either $0 \%$ or $12.5 \%$ ethanol (vol/vol) from $4 \mathrm{~d}$ before mating until GD4 (Fig S2B). Dams had ad libitum access to the liquid diet $21 \mathrm{~h}$ daily and consumed equivalent calories. Water was offered during the remaining $3 \mathrm{~h}$ of the day. On GD5, liquid diets were removed and replaced with standard laboratory chow. On GD20, the placentas were immediately separated into the labyrinth and junctional zone, snap-frozen in liquid nitrogen and stored at $-80^{\circ} \mathrm{C}$ preceding RNA isolation.

\section{Nonhuman primate model of PAE}

As previously described in detail (83), adult female rhesus macaques were trained to orally self-administer either $1.5 \mathrm{~g} / \mathrm{kg} / \mathrm{d}$ of $4 \%$ ethanol solution (equivalent to six drinks/d) or an isocaloric control fluid before time-mated breeding. Each pregnant animal continued ethanol exposure until gestational day 60 (GD60, term gestation is $168 \mathrm{~d}$ in the rhesus macaque) (101). Pregnancies were terminated by cesarean section delivery at three different time points; GD85, GD110, or GD135 (Fig S2C). The macaque placenta is typically bilobed with the umbilical cord insertion in the primary lobe and bridging vessels supplying the fetal side vasculature to the secondary lobe (Fig 2D showing gross placenta anatomy) (102). Full thickness tissue biopsies (maternal decidua to fetal membranes) were taken from both the primary and secondary lobes of the placenta (Fig 2E showing H\&E section of placenta). Samples were immediately snap-frozen in liquid nitrogen and stored at $-80^{\circ} \mathrm{C}$ preceding RNA isolation.

\section{Cell culture trophoblast models}

BeWO human cytotrophoblastic choriocarcinoma cells and HTR8/SVneo extravillous cells were sourced from ATCC (Cat No. CCL-98 and CRL-3271, respectively). BeWO cells were maintained in HAM'S F12 media containing penicillin $(100 \mathrm{U} / \mathrm{ml})$, streptomycin $(100 \mu \mathrm{g} / \mathrm{ml})$, and $10 \%$ vol $/$ vol $\mathrm{FCS}$ at $37^{\circ} \mathrm{C}$ and $5 \% \mathrm{CO}_{2}$. HTR8 cells were maintained in RPMI-1640 media with 5\% vol/vol FCS, under otherwise identical conditions. Culture medium was replenished every $2 \mathrm{~d}$ and cells subcultured every 4-5 d.

BewO cells were treated with $20 \mu \mathrm{M}$ forskolin to induce syncytialization, as previously described $(103,104)$. BeWO and HTR8 cells were also subjected to four separate ethanol treatment conditions: $0 \mathrm{mg} / \mathrm{dl}$, $60 \mathrm{mg} / \mathrm{dl}$ (13 mM), $120 \mathrm{mg} / \mathrm{dl}$ ( $26 \mathrm{mM})$, or $320 \mathrm{mg} / \mathrm{dl}$ (70 mM). To achieve HEa miRNA overexpression and inhibition, Dharmacon miRIDIAN miRNA mimics and hairpin inhibitors (25 nM), or control mimic (Cat No. CN001000-01-05; Dharmacon) and hairpin inhibitor (Cat No. CN-00100001-05; Dharmacon) (25 nm), were transfected into subconfluent BeWO and HTR8 cells using RNAiMAX lipofection reagent (Cat No. 13778; Thermo Fisher Scientific).

\section{Cell cycle analysis}

At $48 \mathrm{~h}$ post transfection, BeWO cells were pulsed with $10 \mu \mathrm{M} \mathrm{EdU}$ for $1 \mathrm{~h}$. The cells were immediately harvested, and cell cycle analysis was performed with the Click-iT EdU Alexa Fluor 488 Flow Cytometry Assay kit (Cat No. C10420; Thermo Fisher Scientific), in conjunction with 7-amino-actinomycin D (Cat No. 00-6993-50; Thermo Fisher Scientific), according to the manufacturer's instructions, using the Beckman Coulter Gallios 2/5/3 flow cytometer. Data were analyzed using Kaluza software (Beckman Coulter).

\section{Cell death analysis}

BeWO cell culture was harvested $48 \mathrm{~h}$ post transfection. Media was subjected to lactate dehydrogenase (LDH) detection using the Pierce LDH Cytotoxicity Assay kit (Cat No. 88953; Thermo Fisher Scientific), according to the manufacturer's instructions, for lytic cell death quantification. The Promega Caspase-Glo 3/7 Assay system (Cat No. G8091; Promega) was used to quantify apoptotic cell death.

\section{Invasion assay}

At $24 \mathrm{~h}$ post-transfection and / or ethanol exposure, HTR8 cells were serum-starved for an additional $18 \mathrm{~h}$. Subsequently, HTR8 cells were seeded onto transwell permeable supports precoated with $300 \mu \mathrm{g} / \mathrm{ml}$ Matrigel (Cat No. 354248; Corning). After 24 h, cells remaining in the apical chamber were removed with a cotton swab. Cells that invaded into the basal chamber were incubated with 1.2 mM 3-(4,5-dimethylthiazol-2-yl)-2,5-diphenyltetrazolium bromide (MTT) for $3 \mathrm{~h}$, and the precipitate solubilized with 10\% SDS in 
$0.01 \mathrm{~N} \mathrm{HCl}$. Absorbance intensities were read at $570 \mathrm{~nm}$ in a Tecan Infinite 200 plate reader.

\section{Metabolic flux analysis and calcium imaging}

BeWO cells (10,000/well) were plated into Seahorse XF96 Cell Culture Microplates (Cat No. 103275-100; Agilent Biotechnology). The OCR, a measure of mitochondrial respiration, and ECAR, a measure of glycolysis, were measured using the Seahorse XFe96 flux analyzer (Seahorse Bioscience). At the time of assay, the cell culture medium was replaced with the appropriate prewarmed Seahorse XF Base Medium (Cat No. 102353-100; Agilent Biotechnology). OCR and ECAR parameters were measured using the Seahorse XFp Cell Energy Phenotype Test kit (Cat No. 103275-100; Agilent Biotechnology). Metabolic stress was induced by simultaneous treatment with $1 \mu \mathrm{m}$ oligomycin and $0.125 \mu \mathrm{M}$ carbonyl cyanide $\mathrm{p}$-[trifluoromethoxy]phenyl-hydrazone (FCCP).

BeWO cells were also plated onto glass coverslips in 24-well plates at a density of 30,000 cells/well. After exposure to ethanol and/or forskolin in culture, the cells were prepared for calcium imaging. After replacement of the culture media with external imaging media ( $154 \mathrm{mM} \mathrm{NaCl}, 5 \mathrm{mM} \mathrm{KCl}, 2 \mathrm{mM} \mathrm{CaCl}, 0.5 \mathrm{mM} \mathrm{MgCl}_{2}$, $5 \mathrm{mM}$ glucose, and $10 \mathrm{mM}$ Hepes, $\mathrm{pH}$ 7.4), the cells were loaded for $35 \mathrm{~min}$ at $37^{\circ} \mathrm{C}$ with the calcium indicator dye fluo-4 AM (Cat No. F14201; Thermo Fisher Scientific), at a final concentration of $5 \mu \mathrm{M}$ fluo-4 AM in 0.1\% DMSO. After incubation, the cells were washed to remove remaining extracellular fluo-4 and imaged at $40 \times$ using confocal microscopy (FV1200-equipped BX61WI microscope; Olympus Corporation). Time-lapse images were acquired at a frequency of $0.5 \mathrm{~Hz}$. Individual cells were manually outlined, and area and mean fluorescence intensity were obtained for each cell (FIJl image processing package) (105). To determine the functional calcium range of each cell, at the end of imaging, the cells were exposed to $5 \mu \mathrm{M}$ ionomycin and $10 \mathrm{mM}$ EGTA (0 mM external $\mathrm{Ca}^{2+}$, $\left.F_{\text {range }}=F_{\text {ionomycin }}-F_{E G T A}\right)$. Baseline fluorescence was determined by averaging the lowest five consecutive fluorescence values during the initial 5 min ( $\left.F_{\text {baseline }}\right)$, which was then expressed as a percentage of $F_{\text {range }}\left(\Delta F_{\text {baseline }}=\left(F_{\text {baseline }}-F_{E G T A}\right) / F_{\text {range }} \times 100\right)$. Maximal intracellular calcium response to $100 \mu \mathrm{M}$ ATP was determined by averaging the highest three consecutive fluorescence values during ATP application $\left(F_{A T P}\right)$ and determining the amount of fluorescence as a percentage of $F_{\text {range }}\left(\Delta F_{\text {ATP }}=\left(F_{\text {ATP }}-F_{E G T A}\right) / F_{\text {range }} \times 100\right)$.

\section{Quantitative reverse transcriptase-polymerase chain reaction analysis}

Total RNA was extracted from tissue, as well as BeWO and HTR8 cells, using the miRNeasy Mini kit (Cat No. 217004; QIAGEN). For miRNA qPCR assays, cDNA was synthesized from $200 \mathrm{ng}$ of total RNA using the miRCURY LNA Universal RT CDNA synthesis kit (Cat No. 203301; Exiqon/Cat No. 339340; QIAGEN), and expression was assessed using miRCURY LNA SYBR Green (Cat No. 203401; Exiqon/ Cat No. 339345; QIAGEN). For mRNA qPCR assays, cDNA was synthesized from $500 \mathrm{ng}$ of total RNA using the qScript cDNA Synthesis kit (Cat No. 95047; Quanta/QIAGEN). Gene expression analysis was performed using PerfeCTa SYBR Green FastMix (Cat No. 95073; Quanta) on the ViiA 7 Real-Time PCR System (Thermo Fisher Scientific). The data presented correspond to the mean $2^{-\Delta \Delta C t}$ after being normalized to the geometric mean of $\beta$-actin, hypoxanthine-guanine phosphoribosyltransferase 1 (HPRT1), and 18s rRNA. Expression data for miRNA was normalized to the geometric mean of miR-25-3p, miR574-3p, miR-30b-5p, miR-652-3p, and miR-15b-5p. For each primer pair, thermal stability curves were assessed for evidence of a single amplicon, and the length of each amplicon was verified using agarose gel electrophoresis. A list of primers and their sequences is presented in Table S3.

\section{Western immunoblotting analysis}

Protein was extracted using 1× RIPA lysis buffer (MilliporeSigma) supplemented with Halt Protease Inhibitor Cocktail (Thermo Fisher Scientific). Tissue was homogenized using the Branson Sonifier 150. Protein concentration was determined using Pierce BCA protein assay kit (Thermo Fisher Scientific), and $30 \mu \mathrm{g}$ of protein was loaded onto a 4\%-12\% Bis-Tris (Cat No. NPO323BOX; Invitrogen/Thermo Fisher Scientific), size-fractionated at $200 \mathrm{~V}$ for $35 \mathrm{~min}$, and transferred to a PVDF membrane using the iBlot transfer system (Invitrogen/Thermo Fisher Scientific). Blots with protein from cultured cells were blocked with 5\% nonfat dry milk in tris-buffered saline containing Tween-20 (TTBS) for $1 \mathrm{~h}$ and incubated overnight with primary antibody. The blot was then washed and incubated with an HRP-conjugated goat anti-rabbit or anti-mouse IgG (Invitrogen) at dilution 1:1,000 for 1-h, then developed using PerkinElmer Western Lightning Plus Chemi ECL (PerkinElmer) and visualized using a CCD camera (Fluorchem Q, Alpha Innotech). Blots with protein from homogenized tissue were dried overnight, rehydrated in methanol, stained with REVERT Total Protein Stain, and developed with the Odyssey CLx Imaging System (LI-COR). Blots were then blocked with Odyssey Blocking Buffer (TBS) for $1 \mathrm{~h}$ and incubated overnight with primary antibody. The blot was then washed and incubated with IRDye 800CW secondary antibody (Cat No. 925-32210; LI-COR). The following antibodies were used: $\beta$-Actin HRP (Cat No. sc-47778; Santa Cruz Biotechnology); Goat anti-Mouse IgG $(\mathrm{H}+\mathrm{L})$ Secondary Antibody, HRP (Cat No. 62-6520; Thermo Fisher Scientific); Goat anti-Rabbit IgG $(\mathrm{H}+\mathrm{L})$ Secondary Antibody, HRP (Cat No. 65-6120; Thermo Fisher Scientific); purified Mouse Anti-E-Cadherin (Cat No. 610181; BD Biosciences); and Rabbit antivimentin antibody (EPR3776) (Cat No. ab 924647; Abcam). Protein levels were quantified using the densitometric analysis package in FIJI image processing software (105).

\section{ELISA}

The second and third trimester maternal plasma samples were collected as part of a longitudinal cohort study conducted in two regions of Western Ukraine as part of the Collaborative Initiative on FASDs (CIFASD.org) between the years 2006 and 2011, as previously reported (8). Plasma, at a 1:1,000 dilution, was subjected to hCG detection using Abcam's intact human hCG ELISA kit (Cat no. ab100533) following the manufacturer's protocol. 


\section{Literature review}

We conducted a literature review for HEamiRNAs and their associated gestational pathology using the National Institute of Health's PubMed search interface. For each miRNA, the following search parameters were used:

\section{[ MIRX OR MIR X OR MIRNA X OR MIRNAX or MIRNX]}

$$
\text { AND MeSH Term }
$$

where $X$ represents the miRNA of interest and automatic term expansion was enabled. The following MeSH terms, and related search terms (in brackets), were used: Fetal Growth Retardation (Intrauterine Growth Retardation, IUGR, Intrauterine Growth Restriction, Low Birth Weight, LBW, Small For Gestational Age, SGA), Premature Birth (Preterm Birth, Preterm Birth, Preterm Infant, Premature Infant, Preterm Labor, Premature Labor), Spontaneous Abortion (Early Pregnancy Loss, Miscarriage, Abortion, Tubal Abortion, Aborted Fetus), Pre-Eclampsia (Pre Eclampsia, Preeclampsia, Pregnancy Toxemia, Gestational Hypertension, Maternal Hypertension), and Maternal Exposure (Environmental Exposure, Prenatal Exposure). Returned articles were subsequently assessed for relevance.

\section{Secondary analysis of RNA sequencing data}

Expression levels of ${ }_{\mathrm{HEa}}$ miRNAs in tissues were determined using the Human miRNA Expression Database and the miRmine Human miRNA expression database $(58,106)$. For expression analysis of HEamiRNA pri-miRNAs, RNA sequencing data were used from NCBI's sequence read archive (https://www.ncbi.nlm.nih.gov/sra). The accession numbers for the sequence files are uterus (SRR1957209), thyroid (SRR1957207), thymus (SRR1957206), stomach (SRR1957205), spleen (SRR1957203), small intestine (SRR1957202), skeletal muscle (SRR1957201), salivary gland (SRR1957200), placenta (SRR1957197), lung (SRR1957195), liver (SRR1957193), kidney (SRR1957192), heart (SRR1957191), whole brain (SRR1957183), adrenal gland (SRR1957124), bone marrow (ERR315396), colon (ERR315484), adipose tissue (ERR315332), and pancreas (ERR315479). Deep sequencing analysis was conducted using the Galaxy version 15.07 user interface according to the bioinformatics pipeline outlined in Fig S12.

\section{Statistical analyses}

Linear regression models were used to estimate associations between infant growth measures and miRNA expression levels, gestational age at blood draw, the interaction between subjectcentered miRNA expression level and gestational age at blood draw, and child sex. Spearman correlations between infant growth measures and subject-centered miRNA expression levels were also calculated. Linear regression models were also used to estimate the associations between gestational at birth and logtransformed hCG levels, ethanol intake, the interaction between log-transformed hCG levels and ethanol intake, gestational at blood draw, and child sex. Statistical analysis and graphs were generated with GraphPad Prism 6 software (GraphPad Software, Inc), SPSS v24, or R version 3.3.1. Results are expressed as the mean \pm SEM or alternatively as box and whisker plots with the bounds of the box demarcating limits of first and third quartile, a median line in the center of the box, and whiskers representing the total range of data. The overall group effect was analyzed for significance using one-way MANOVA, one-way or two-way ANOVA with Tukey's HSD or Dunnett's multiple comparisons post hoc testing when appropriate (i.e., following a significant group effect in one-way ANOVA or given a significant interaction effect between experimental conditions in two-way ANOVA), to correct for a family-wise error rate. A two-tailed $t$ test was used for planned comparisons. For experiments characterizing the in-

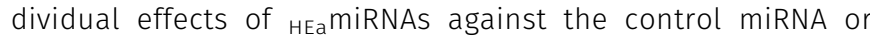
antagomirs, individual two-tailed t test with 5\% FDR correction was applied to account for multiple comparisons. All statistical tests, sample sizes, and post hoc analysis are appropriately reported in the results section. A value of $P<0.05$ was considered statistically significant and a value of $0.1<P<0.05$ was considered marginally significant.

\section{Study approval}

Human study protocols were approved by the institutional review boards at the Lviv National Medical University, Ukraine, and the University of California San Diego as well as Texas A\&M University in the United States. Research was conducted according to the principles expressed in the Declaration of Helsinki with written informed consent received from participants before inclusion in the study. All rodent experiments were performed in accordance with protocols approved by the University of New Mexico Institutional Animal Care and Use Committee (IACUC), the Texas A\&M University IACUC, and the University of Queensland Animal Ethics Committees. All procedures involving nonhuman primate research subjects were approved by the IACUC of the Oregon National Primate Research Center (ONPRC), and guidelines for humane animal care were followed. The ONPRC abides by the Animal Welfare Act and Regulations enforced by the US Department of Agriculture.

\section{Supplementary Information}

Supplementary Information is available at https://doi.org/10.26508/Isa. 201800252.

\section{Acknowledgements}

This research was supported by grants from the NIH, P50 AA022534 (AM Allan), U01 AA014835 and the Office of Dietary Supplements (CD Chambers), R24 AA019431 (KA Grant), R01 AA021981 (CD Kroenke), R01 AA024659 (RC Miranda), and F31 AA026505 (AM Tseng). We thank the National Health and Medical Research Council of Australia (KM Moritz) for their support. We thank CIFASD for intellectual support and Megan S Pope and Tenley E Lehman for their assistance in conducting cell culture and animal studies. Data on human subjects are deposited at CIFASD.org, in accordance with NIH data repository guidelines. 


\section{Author Contributions}

AM Tseng: investigation, methodology, and writing-original draft, review, and editing.

AH Mahnke: investigation, methodology, and writing-review and editing.

AB Wells: formal analysis and writing-review and editing.

NA Salem: methodology and writing-review and editing.

AM Allan: investigation, methodology, and writing-review and editing.

VHJ Roberts: investigation, methodology, and writing-review and editing.

N Newman: investigation, methodology, and writing-review and editing.

NAR Walter: investigation, methodology, and writing-review and editing.

CD Kroenke: investigation, methodology, and writing-review and editing.

KA Grant: investigation, methodology, and writing-review and editing.

LK Akison: investigation, methodology, and writing-review and editing.

KM Moritz: investigation, methodology, and writing-review and editing.

CD Chambers: supervision, investigation, methodology, and writing-review and editing.

RC Miranda: supervision, investigation, methodology, and writing-review and editing.

\section{Conflict of Interest Statement}

The authors declare that they have no conflict of interest.

\section{References}

1. Popova S, Lange S, Probst C, Gmel G, Rehm J (2017) Estimation of national, regional, and global prevalence of alcohol use during pregnancy and fetal alcohol syndrome: A systematic review and metaanalysis. Lancet Glob Health 5: e290-e299. doi:10.1016/s2214-109x(17) 30021-9

2. SAMHSA. The NSDUH Report: 18 Percent of Pregnant Women Drink Alcohol during Early Pregnancy. NSDUH Report. 2013.

3. Bakhireva LN, Sharkis J, Shrestha S, Miranda-Sohrabji TJ, Williams S, Miranda RC (2017) Prevalence of prenatal alcohol exposure in the state of Texas as assessed by phosphatidylethanol in newborn dried blood spot specimens. Alcohol Clin Exp Res 41: 1004-1011. doi:10.1111/ acer.13375

4. May PA, Chambers CD, Kalberg WO, Zellner J, Feldman H, Buckley D, Kopald D, Hasken JM, Xu R, Honerkamp-Smith G, et al (2018) Prevalence of fetal alcohol spectrum disorders in 4 US communities. JAMA 319: 474-482. doi:10.1001/jama.2017.21896

5. Roozen S, Peters GJ, Kok G, Townend D, Nijhuis J, Curfs L (2016) Worldwide prevalence of fetal alcohol spectrum disorders: A systematic literature review including meta-analysis. Alcohol Clin Exp Res 40: 18-32. doi:10.1111/acer.12939

6. Lange S, Rehm J, Anagnostou E, Popova S (2017) Prevalence of externalizing disorders and autism spectrum disorders among children with fetal alcohol spectrum disorder: Systematic review and meta-analysis. Biochem Cell Biol 1-11. doi:10.1139/bcb-2017-0014

7. Bertrand J, Floyd RL, Weber MK, O'Conner M, Johnson KA, Riley EP, Cohen $D E$. National task force on fetal alcohol syndrome and fetal alcohol effect. In: US Department of Health and Human Services. Atlanta, GA: CDC; 2004.

8. Balaraman S, Schafer JJ, Tseng AM, Wertelecki W, Yevtushok L, ZymakZakutnya N, Chambers CD, Miranda RC (2016) Plasma miRNA profiles in pregnant women predict infant outcomes following prenatal alcohol exposure. PLoS One 11: e0165081. doi:10.1371/journal.pone.0165081

9. Rossant J, Cross JC (2001) Placental development: Lessons from mouse mutants. Nat Rev Genet 2: 538. doi:10.1038/35080570

10. E Davies J, Pollheimer J, Yong HEJ, Kokkinos MI, Kalionis B, Knöfler M, Murthi P (2016) Epithelial-mesenchymal transition during extravillous trophoblast differentiation. Cell Adhes Migration 10: 310-321. doi:10.1080/19336918.2016.1170258

11. Zhou Y, Damsky CH, Fisher SJ (1997) Preeclampsia is associated with failure of human cytotrophoblasts to mimic a vascular adhesion phenotype. One cause of defective endovascular invasion in this syndrome? J Clin Invest 99: 2152-2164. doi:10.1172/jci119388

12. Damsky CH, Fisher SJ (1998) Trophoblast pseudo-vasculogenesis: Faking it with endothelial adhesion receptors. Curr Opin Cel Biol 10: 660-666. doi:10.1016/s0955-0674(98)80043-4

13. Brown LM, Lacey HA, Baker PN, Crocker IP (2005) E-cadherin in the assessment of aberrant placental cytotrophoblast turnover in pregnancies complicated by pre-eclampsia. Histochem Cell Biol 124: 499-506. doi:10.1007/s00418-005-0051-7

14. Fedorova L, Gatto-Weis C, Smaili S, Khurshid N, Shapiro JI, Malhotra D, Horrigan T (2012) Down-regulation of the transcription factor snail in the placentas of patients with preeclampsia and in a rat model of preeclampsia. Reprod Biol Endocrinol 10: 15. doi:10.1186/1477-7827-10-15

15. Blechschmidt K, Mylonas I, Mayr D, Schiessl B, Schulze S, Becker KF, Jeschke U (2007) Expression of E-cadherin and its repressor snail in placental tissue of normal, preeclamptic and HELLP pregnancies. Virchows Archiv 450: 195-202. doi:10.1007/s00428-006-0343-x

16. Du L, Kuang L, He F, Tang W, Sun W, Chen D (2017) Mesenchymal-toepithelial transition in the placental tissues of patients with preeclampsia. Hypertens Res 40: 67-72. doi:10.1038/hr.2016.97

17. Gundogan F, Gilligan J, Ooi JH, Sung J, Qi W, Naram R, de la Monte SM (2013) Dual mechanisms of ethanol-impaired placentation: Experimental model. J Clin Exp Pathol 3: 142. doi:10.4172/21610681.1000142.

18. Tai M, Piskorski A, Kao JCW, Hess LA, M de la Monte S, Gündoğan F (2017) Placental morphology in fetal alcohol spectrum disorders. Alcohol Alcohol 52: 138-144. doi:10.1093/alcalc/agw088

19. Gundogan F, Gilligan J, Qi W, Chen E, Naram R, de la Monte SM (2015) Dose effect of gestational ethanol exposure on placentation and fetal growth. Placenta 36: 523-530. doi:10.1016/j.placenta.2015.02.010

20. Gardebjer EM, Cuffe JS, Pantaleon M, Wlodek ME, Moritz KM (2014) Periconceptional alcohol consumption causes fetal growth restriction and increases glycogen accumulation in the late gestation rat placenta. Placenta 35: 50-57. doi:10.1016/j.placenta.2013.10.008

21. Kalisch-Smith JI, Outhwaite JE, Simmons DG, Pantaleon M, Moritz KM (2016) Alcohol exposure impairs trophoblast survival and alters subtype-specific gene expression in vitro. Placenta 46: 87-91. doi:10.1016/j.placenta.2016.08.080

22. Bahado-Singh RO, Oz AU, Kingston JM, Shahabi S, Hsu CD, Cole L (2002) The role of hyperglycosylated hCG in trophoblast invasion and the prediction of subsequent pre-eclampsia. Prenatal Diagn 22: 478-481. doi:10.1002/pd.329

23. Muller F, Savey L, Le Fiblec B, Bussieres L, Ndayizamba G, Colau JC, Giraudet P (1996) Maternal serum human chorionic gonadotropin level 
at fifteen weeks is a predictor for preeclampsia. Am J Obstet Gynecol 175: 37-40. doi:10.1016/s0002-9378(96)70247-8

24. Spencer K, Macri JN, Aitken DA, Connor JM (1992) Free beta-hCG as firsttrimester marker for fetal trisomy. Lancet (London, England) 339: 1480. doi:10.1016/0140-6736(92)92073-0

25. Spencer K (1991) Evaluation of an assay of the free beta-subunit of choriogonadotropin and its potential value in screening for Down's syndrome. Clin Chem 37: 809-814.

26. Salihu HM, Kornosky JL, Lynch O, Alio AP, August EM, Marty PJ (2011) Impact of prenatal alcohol consumption on placenta-associated syndromes. Alcohol 45: 73-79. doi:10.1016/j.alcohol.2010.05.010

27. Khong TY (2004) Placental vascular development and neonatal outcome. Semin Neonatal 9: 255-263. doi:10.1016/j.siny.2003.11.010

28. Ray JG, Vermeulen MJ, Schull MJ, Redelmeier DA (2005) Cardiovascular health after maternal placental syndromes (CHAMPS): Populationbased retrospective cohort study. Lancet (London, England) 366: 1797-1803. doi:10.1016/s0140-6736(05)67726-4

29. Wang D, Na Q, Song WW, Song GY (2014) Altered Expression of miR-518b and miR-519a in the placenta is associated with low fetal birth weight. Am J Perinatol 31: 729-734. doi:10.1055/s-0033-1361832

30. Wang JM, Gu Y, Zhang Y, Yang Q, Zhang X, Yin L, Wang J (2016) Deepsequencing identification of differentially expressed miRNAs in decidua and villus of recurrent miscarriage patients. Arch Gynecol Obstetrics 293: 1125-1135. doi:10.1007/s00404-016-4038-5

31. Hromadnikova I, Kotlabova K, Ondrackova M, Pirkova P, Kestlerova A, Novotna V, Hympanova L, Krofta L (2015) Expression profile of C19MC microRNAs in placental tissue in pregnancy-related complications. DNA Cell Biol 34: 437-457. doi:10.1089/dna.2014.2687

32. Hromadnikova I, Kotlabova K, Ivankova K, Krofta L (2017) Expression profile of C19MC microRNAs in placental tissue of patients with preterm prelabor rupture of membranes and spontaneous preterm birth. Mol Med Rep 16: 3849-3862. doi:10.3892/mmr.2017.7067

33. Timofeeva AV, Gusar VA, Kan NE, Prozorovskaya KN, Karapetyan AO, Bayev OR, Chagovets VV, Kliver SF, Iakovishina DY, Frankevich VE, et al (2018) Identification of potential early biomarkers of preeclampsia. Placenta 61: 61-71. doi:10.1016/j.placenta.2017.11.011

34. Dong F, Zhang Y, Xia F, Yang Y, Xiong S, Jin L, Zhang J (2014) Genome-wide miRNA profiling of villus and decidua of recurrent spontaneous abortion patients. Reproduction (Cambridge, England) 148: 33-41. doi:10.1530/rep-14-0095

35. Hu Y, Li P, Hao S, Liu L, Zhao J, Hou Y (2009) Differential expression of microRNAs in the placentae of Chinese patients with severe pre-eclampsia. Clin Chem Lab Med 47: 923-929. doi:10.1515/cclm.2009.228

36. Murphy MS, Casselman RC, Tayade C, Smith GN (2015) Differential expression of plasma microRNA in preeclamptic patients at delivery and 1 year postpartum. Am J Obstet Gynecol 213: 367. e1-e9. doi:10.1016/ j.ajog.2015.05.013

37. Bidarimath M, Edwards AK, Wessels JM, Khalaj K, Kridli RT, Tayade C (2015) Distinct microRNA expression in endometrial lymphocytes, endometrium, and trophoblast during spontaneous porcine fetal loss. J Reprod Immunol 107: 64-79. doi:10.1016/j.jri.2014.11.004

38. Liu XD, Wu X, Yin YL, Liu YQ, Geng MM, Yang HS, Blachier F, Wu GY (2012) Effects of dietary L-arginine or N-carbamylglutamate supplementation during late gestation of sows on the miR-15b/16, miR-221/222, VEGFA and eNOS expression in umbilical vein. Amino Acids 42: 2111-2119. doi:10.1007/s00726-011-0948-5

39. Baker BC, Mackie FL, Lean SC, Greenwood SL, Heazell AEP, Forbes K, Jones RL (2017) Placental dysfunction is associated with altered microRNA expression in pregnant women with low folate status. Mol Nutr Food Res 61. doi:10.1002/mnfr.201600646

40. Gao Y, She R, Wang Q, Li Y, Zhang H (2018) Up-regulation of miR-299 suppressed the invasion and migration of HTR-8/SVneo trophoblast cells partly via targeting HDAC2 in pre-eclampsia. Biomed Pharmacother 97: 1222-1228. doi:10.1016/j.biopha.2017.11.053

41. Sandrim VC, Luizon MR, Palei AC, Tanus-Santos JE, Cavalli RC (2016) Circulating microRNA expression profiles in pre-eclampsia: Evidence of increased mir-885-5p levels. BJOG 123: 2120-2128. doi:10.1111/14710528.13903

42. Rodosthenous RS, Burris HH, Sanders AP, Just AC, Dereix AE, Svensson $K_{\text {, }}$ Solano M, Tellez-Rojo MM, Wright RO, Baccarelli AA (2017) Second trimester extracellular microRNAs in maternal blood and fetal growth: An exploratory study. Epigenetics 12: 804-810. doi:10.1080/ 15592294.2017.1358345

43. Martinez-Fierro ML, Garza-Veloz I, Gutierrez-Arteaga C, Delgado-Enciso I, Barbosa-Cisneros OY, Flores-Morales V, Hernandez-Delgadillo GP, Rocha-Pizana MR, Rodriguez-Sanchez IP, Badillo-Almaraz Jl, et al (2018) Circulating levels of specific members of chromosome 19 microRNA cluster are associated with preeclampsia development. Arch Gynecol Obstet 297: 365-371. doi:10.1007/s00404-017-4611-6

44. Yang S, Li H, Ge Q, Guo L, Chen F (2015) Deregulated microRNA species in the plasma and placenta of patients with preeclampsia. Mol Med Rep 12: 527-534. doi:10.3892/mmr.2015.3414

45. Hromadnikova I, Kotlabova K, Ivankova K, Krofta L (2017) First trimester screening of circulating C19MC microRNAs and the evaluation of their potential to predict the onset of preeclampsia and IUGR. PLoS One 12: e0171756. doi:10.1371/journal.pone.0171756

46. Zhang M, Muralimanoharan S, Wortman AC, Mendelson CR (2016) Primate-specific miR-515 family members inhibit key genes in human trophoblast differentiation and are upregulated in preeclampsia. Proc Natl Acad Sci USA 113: E7069-E76. doi:10.1073/pnas.1607849113

47. Nemoto T, Kakinuma Y, Shibasaki T (2015) Impaired miR449a-induced downregulation of Crhr1 expression in low-birth-weight rats. J Endocrinol 224: 195-203. doi:10.1530/joe-14-0537

48. Mei Z, Huang B, Mo Y, Fan J (2017) An exploratory study into the role of miR-204-5p in pregnancy-induced hypertension. Exp Ther Med 13: 1711-1718. doi:10.3892/etm.2017.4212

49. Choi SY, Yun J, Lee OJ, Han HS, Yeo MK, Lee MA, Suh KS (2013) MicroRNA expression profiles in placenta with severe preeclampsia using a PNAbased microarray. Placenta 34: 799-804. doi:10.1016/j. placenta.2013.06.006

50. Kaufmann P, Black S, Huppertz B (2003) Endovascular trophoblast invasion: Implications for the pathogenesis of intrauterine growth retardation and preeclampsia. Biol Reprod 69: 1-7. doi:10.1095/ biolreprod.102.014977

51. Barrientos G, Pussetto M, Rose M, Staff AC, Blois SM, Toblli JE (2017) Defective trophoblast invasion underlies fetal growth restriction and preeclampsia-like symptoms in the stroke-prone spontaneously hypertensive rat. Mol Hum Reprod 23: 509-519. doi:10.1093/molehr/ gax024

52. Roberts JM, Escudero C (2012) The placenta in preeclampsia. Pregnancy Hypertens 2: 72-83. doi:10.1016/j.preghy.2012.01.001

53. Fisher SJ (2015) Why is placentation abnormal in preeclampsia? Am J Obstet Gynecol 213: S115-S22. doi:10.1016/j.ajog.2015.08.042

54. Crosley EJ, Elliot MG, Christians JK, Crespi BJ (2013) Placental invasion, preeclampsia risk and adaptive molecular evolution at the origin of the great apes: Evidence from genome-wide analyses. Placenta 34: 127-132. doi:10.1016/j.placenta.2012.12.001

55. Lyall F, Bulmer JN, Duffie E, Cousins F, Theriault A, Robson SC (2001) Human trophoblast invasion and spiral artery transformation: The role of PECAM-1 in normal pregnancy, preeclampsia, and fetal growth restriction. Am J Pathol 158: 1713-1721. doi:10.1016/s0002-9440(10)64127-2

56. Goldman-Wohl D, Yagel S (2002) Regulation of trophoblast invasion: From normal implantation to pre-eclampsia. Mol Cell Endocrinol 187: 233-238. doi:10.1016/s0303-7207(01)00687-6 
57. Balaraman S, Lunde ER, Sawant O, Cudd TA, Washburn SE, Miranda RC (2014) Maternal and neonatal plasma microRNA biomarkers for fetal alcohol exposure in an ovine model. Alcohol Clin Exp Res 38: 1390-1400. doi:10.1111/acer.12378

58. Panwar B, Omenn GS, Guan Y (2017) miRmine: a database of human miRNA expression profiles. Bioinformatics 33: 1554-1560. doi:10.1093/ bioinformatics/btx019

59. Vicovac L, Aplin JD (1996) Epithelial-mesenchymal transition during trophoblast differentiation. Acta Anatomica 156: 202-216.

60. Knöfler M, Pollheimer J (2013) Human placental trophoblast invasion and differentiation: A particular focus on wnt signaling. Front Genet 4: 190. doi:10.3389/fgene.2013.00190

61. Arimoto-Ishida E, Sakata M, Sawada K, Nakayama M, Nishimoto F, Mabuchi S, Takeda T, Yamamoto T, Isobe A, Okamoto Y, et al (2009) Upregulation of alpha5-integrin by E-cadherin loss in hypoxia and its key role in the migration of extravillous trophoblast cells during early implantation. Endocrinology 150: 4306-4315. doi:10.1210/en.2008-1662

62. Sun YY, Lu M, Xi XW, Qiao QQ, Chen LL, Xu XM, Feng YJ (2011) Regulation of epithelial-mesenchymal transition by homeobox gene DLX4 in JEG-3 trophoblast cells: A role in preeclampsia. Reprod Sci 18: 1138-1145. doi:10.1177/1933719111408112

63. Barrak J, Msheik H, Abou-Kheir W, Daoud G (2016) Assessment of different trophoblast cell lines as in vitro models for placental development. Placenta 45: 106. doi:10.1016/j.placenta.2016.06.157

64. Lovisa S, LeBleu VS, Tampe B, Sugimoto H, Vadnagara K, Carstens JL, Wu CC, Hagos Y, Burckhardt BC, Pentcheva-Hoang T, et al (2015) Epithelialto-mesenchymal transition induces cell cycle arrest and parenchymal damage in renal fibrosis. Nat Med 21: 998-1009. doi:10.1038/nm.3902

65. Vega S, Morales AV, Ocana OH, Valdes F, Fabregat I, Nieto MA (2004) Snail blocks the cell cycle and confers resistance to cell death. Genes Dev 18: 1131-1143. doi:10.1101/gad.294104

66. Moreau R, Hamel A, Daoud G, Simoneau L, Lafond I (2002) Expression of calcium channels along the differentiation of cultured trophoblast cells from human term placenta. Biol Reprod 67: 1473-1479. doi:10.1095/ biolreprod.102.005397

67. Lu X, He Y, Zhu C, Wang H, Chen S, Lin HY (2016) Twist1 is involved in trophoblast syncytialization by regulating GCM1. Placenta 39: 45-54. doi:10.1016/j.placenta.2016.01.008

68. Omata W, Ackerman WEIV, Vandre DD, Robinson JM (2013) Trophoblast cell fusion and differentiation are mediated by both the protein kinase $C$ and A pathways. PLoS One 8: e81003. doi:10.1371/journal.pone.0081003

69. Spaans F, Melgert BN, Chiang C, Borghuis T, Klok PA, de Vos P, van Goor H, Bakker WW, Faas MM (2014) Extracellular ATP decreases trophoblast invasion, spiral artery remodeling and immune cells in the mesometrial triangle in pregnant rats. Placenta 35: 587-595. doi:10.1016/j.placenta.2014.05.013

70. Karl PI, Chusid J, Tagoe C, Fisher SE (1997) Ca2+ flux in human placental trophoblasts. Am J Physiol 272: C1776-C1780. doi:10.1152/ ajpcell.1997.272.6.c1776

71. Roberts VH, Waters LH, Powell T (2007) Purinergic receptor expression and activation in first trimester and term human placenta. Placenta 28 : 339-347. doi:10.1016/j.placenta.2006.04.007

72. Halmesmaki E, Autti I, Granstrom ML, Stenman UH, Ylikorkala O (1987) Estradiol, estriol, progesterone, prolactin, and human chorionic gonadotropin in pregnant women with alcohol abuse. J Clin Endocrinol Metab 64: 153-156. doi:10.1210/jcem-64-1-153

73. Edelstam G, Karlsson C, Westgren M, Lowbeer C, Swahn ML (2007) Human chorionic gonadatropin (hCG) during third trimester pregnancy. Scand J Clin Lab Invest 67: 519-525. doi:10.1080/ 00365510601187765

74. Soares MJ, Chakraborty D, Rumi MAK, Konno T, Renaud SJ (2012) Rat placentation: An experimental model for investigating the hemochorial maternal-fetal interface. Placenta 33: 233-243. doi:10.1016/j. placenta.2011.11.026

75. Grigsby PL (2016) Animal models to study placental development and function throughout normal and dysfunctional human pregnancy. Semin Reprod Med 34: 11-16. doi:10.1055/s-0035-1570031

76. Vercruysse L, Caluwaerts S, Luyten C, Pijnenborg R (2006) Interstitial trophoblast invasion in the decidua and mesometrial triangle during the last third of pregnancy in the rat. Placenta 27: 22-33. doi:10.1016/j. placenta.2004.11.004

77. Silva JF, Serakides R (2016) Intrauterine trophoblast migration: A comparative view of humans and rodents. Cell Adhes Migration 10: 88-110. doi:10.1080/19336918.2015.1120397

78. Kurtzman JT, Wilson H, Rao CV (2001) A proposed role for hCG in clinical obstetrics. Semin Reprod Med 19: 063-068. doi:10.1055/s-2001-13912

79. Furcron A-E, Romero R, Mial TN, Balancio A, Panaitescu B, Hassan SS, Sahi A, Nord C, Gomez-Lopez N (2016) Human chorionic gonadotropin has anti-inflammatory effects at the maternal-fetal interface and prevents endotoxin-induced preterm birth, but causes dystocia and fetal compromise in mice. Biol Reprod 94: 136. doi:10.1095/ biolreprod. 116.139345

80. Henderson GI, Hoyumpa AM Jr, McClain C, Schenker S (1979) The effects of chronic and acute alcohol administration on fetal development in the rat. Alcohol Clin Exp Res 3: 99-106. doi:10.1111/j.1530-0277.1979. tb05281.x

81. O'Leary-Moore SK, Parnell SE, Godin EA, Dehart DB, Ament JJ, Khan AA, Johnson GA, Styner MA, Sulik KK (2010) Magnetic resonance microscopy-based analyses of the brains of normal and ethanolexposed fetal mice. Birth Defects Res A Clin Mol Teratol 88: 953-964. doi:10.1002/bdra.20719

82. Bake S, Tingling JD, Miranda RC (2012) Ethanol exposure during pregnancy persistently attenuates cranially directed blood flow in the developing fetus: Evidence from ultrasound imaging in a murine second trimester equivalent model. Alcohol Clin Exp Res 36: 748-758. doi:10.1111/j.1530-0277.2011.01676.x

83. Lo JO, Schabel MC, Roberts VH, Wang X, Lewandowski KS, Grant KA, Frias $A E$, Kroenke CD (2017) First trimester alcohol exposure alters placental perfusion and fetal oxygen availability affecting fetal growth and development in a non-human primate model. Am J Obstet Gynecol 216: 302. e1-e8. doi:10.1016/j.ajog.2017.01.016

84. Xie L, Mouillet J-F, Chu T, Parks WT, Sadovsky E, Knöfler M, Sadovsky Y (2014) C19MC MicroRNAs regulate the migration of human trophoblasts. Endocrinology 155: 4975-4985. doi:10.1210/en.2014-1501

85. Smith SM, Garic A, Flentke GR, Berres ME (2014) Neural crest development in fetal alcohol syndrome. Birth Defects Res C Embryo Today 102: 210-220. doi:10.1002/bdrc.21078

86. Kalcheim C (2016) Epithelial-mesenchymal transitions during neural crest and somite development. Journal Clin Med 5: 1. doi:10.3390/jcm5010001

87. Yang J, Qiu H, Qu P, Zhang R, Zeng L, Yan H (2015) Prenatal alcohol exposure and congenital heart defects: A meta-analysis. PLoS One 10: e0130681. doi:10.1371/journal.pone. 0130681

88. Burd L, Deal E, Rios R, Adickes E, Wynne J, Klug MG (2007) Congenital heart defects and fetal alcohol spectrum disorders. Congenit Heart Dis 2: 250-255. doi:10.1111/j.1747-0803.2007.00105.x

89. Serrano M, Han M, Brinez P, Linask KK (2010) Fetal alcohol syndrome: Cardiac birth defects in mice and prevention with folate. Am J Obstet Gynecol 203: 75. e7-e15. doi:10.1016/j.ajog.2010.03.017

90. Sarmah S, Marrs JA (2013) Complex cardiac defects after ethanol exposure during discrete cardiogenic events in zebrafish: Prevention with folic acid. Dev Dyn 242: 1184-1201. doi:10.1002/dvdy.24015

91. Combs MD, Yutzey KE (2013) Heart valve development: Regulatory networks in development and disease. Circ Res 105: 408-421. doi:10.1161/circresaha.109.201566 
92. Lin C-J, Lin C-Y, Chen C-H, Zhou B, Chang C-P (2012) Partitioning the heart: Mechanisms of cardiac septation and valve development. Development (Cambridge, England) 139: 3277-3299. doi:10.1242/dev.063495

93. Janssen HL, Reesink HW, Lawitz EJ, Zeuzem S, Rodriguez-Torres M, Patel K, van der Meer AJ, Patick AK, Chen A, Zhou Y, et al (2013) Treatment of HCV infection by targeting microRNA. New Engl J Med 368: 1685-1694. doi:10.1056/nejmoa1209026

94. Beg MS, Brenner AJ, Sachdev J, Borad M, Kang YK, Stoudemire J, Smith S, Bader AG, Kim S, Hong DS (2017) Phase I study of MRX34, a liposomal miR34a mimic, administered twice weekly in patients with advanced solid tumors. Invest New Drugs 35: 180-188. doi:10.1007/s10637-016-0407-y

95. Sathyan P, Golden HB, Miranda RC (2007) Competing interactions between micro-RNAs determine neural progenitor survival and proliferation after ethanol exposure: Evidence from an ex vivo model of the fetal cerebral cortical neuroepithelium. J Neurosci 27: 8546-8557. doi:10.1523/jneurosci.1269-07.2007

96. Huang W, Tian SS, Hang PZ, Sun C, Guo J, Du ZM (2016) Combination of microRNA-21 and microRNA-146a attenuates cardiac dysfunction and apoptosis during acute myocardial infarction in mice. Mol Ther Nucleic Acids 5: e296. doi:10.1038/mtna.2016.12

97. Brady ML, Allan AM, Caldwell KK (2012) A limited access mouse model of prenatal alcohol exposure that produces long-lasting deficits in hippocampal-dependent learning and memory. Alcohol Clin Exp Res 36: 457-466. doi:10.1111/j.1530-0277.2011.01644.x

98. Kajimoto K, Allan A, Cunningham LA (2013) Fate analysis of adult hippocampal progenitors in a murine model of fetal alcohol spectrum disorder (FASD). PLoS One 8: e73788. doi:10.1371/journal. pone. 0073788

99. Watson ED, Cross JC (2005) Development of structures and transport functions in the mouse placenta. Physiology (Bethesda) 20: 180-193. doi:10.1152/physiol.00001.2005

100. Gårdebjer EM, Anderson ST, Pantaleon M, Wlodek ME, Moritz KM (2015) Maternal alcohol intake around the time of conception causes glucose intolerance and insulin insensitivity in rat offspring, which is exacerbated by a postnatal high-fat diet. FASEB J 29: 2690-2701. doi:10.1096/fj.14-268979

101. Grant KA, Leng X, Green HL, Szeliga KT, Rogers LS, Gonzales SW (2008) Drinking typography established by scheduled induction predicts chronic heavy drinking in a monkey model of ethanol self-administration. Alcohol Clin Exp Res 32: 1824-1838. doi:10.1111/ j.1530-0277.2008.00765.x

102. Carter AM (2007) Animal models of human placentation: A review. Placenta 28 Suppl A:S41-S47. doi:10.1016/j. placenta.2006.11.002

103. Orendi K, Gauster M, Moser G, Meiri H, Huppertz B (2010) The choriocarcinoma cell line BeWo: Syncytial fusion and expression of syncytium-specific proteins. Reproduction (Cambridge, England) 140: 759-766. doi:10.1530/rep-10-0221

104. Oh SY, Hwang JR, Lee Y, Choi SJ, Kim JS, Kim JH, Sadovsky Y, Roh CR (2016) Isolation of basal membrane proteins from BeWo cells and their expression in placentas from fetal growth-restricted pregnancies. Placenta 39: 24-32. doi:10.1016/ j.placenta.2016.01.001

105. Schindelin J, Arganda-Carreras I, Frise E, Kaynig V, Longair M, Pietzsch T, Preibisch S, Rueden C, Saalfeld S, Schmid B, et al (2012) Fiji: An opensource platform for biological-image analysis. Nat Methods 9: 676-682. doi:10.1038/nmeth.2019

106. Gong J, Wu Y, Zhang X, Liao Y, Sibanda VL, Liu W, Guo AY (2014) Comprehensive analysis of human small RNA sequencing data provides insights into expression profiles and miRNA editing. RNA Biol 11: 1375-1385. doi:10.1080/ 15476286.2014.996465

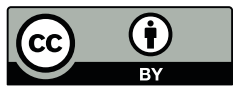

License: This article is available under a Creative Commons License (Attribution 4.0 International, as described at https://creativecommons.org/ licenses/by/4.0/). 